\title{
A NUMERICAL METHOD FOR COMPUTING AN SVD-LIKE DECOMPOSITION*
}

\author{
HONGGUO XU ${ }^{\dagger}$
}

\begin{abstract}
We present a numerical method for computing the SVD-like decomposition $B=$ $Q D S^{-1}$, where $Q$ is orthogonal, $S$ is symplectic, and $D$ is a permuted diagonal matrix. The method can be applied directly to compute the canonical form of the Hamiltonian matrices of the form $J B^{T} B$, where $J=\left[\begin{array}{cc}0 & I \\ -I & 0\end{array}\right]$. It can also be applied to solve the related application problems such as the gyroscopic systems and linear Hamiltonian systems. Error analysis and numerical examples show that the eigenvalues of $J B^{T} B$ computed by this method are more accurate than those computed by the methods working on the explicit product $J B^{T} B$ or $B J B^{T}$.
\end{abstract}

Key words. skew-symmetric matrix, Hamiltonian matrix, symplectic matrix, orthogonal symplectic matrix, eigenvalue problem, SVD, SVD-like decomposition, Schur form, Jordan canonical form, QR algorithm, Jacobi algorithm

AMS subject classification. $65 \mathrm{~F} 15$

DOI. $10.1137 /$ S0895479802410529

1. Introduction. It is shown in [18] that every real matrix $B \in \mathbb{R}^{n \times 2 m}$ has an SVD-like decomposition

$$
\left.Q^{T} B S=\begin{array}{lrcccc}
p & q & m-p-q & p & q & m-p-q \\
p & \\
p & \\
n-2 p-q & 0 & 0 & 0 & 0 & 0 \\
0 & I & 0 & 0 & 0 & 0 \\
0 & 0 & 0 & \Sigma & 0 & 0 \\
0 & 0 & 0 & 0 & 0 & 0
\end{array}\right),
$$

where matrix $Q$ is real orthogonal, $S$ is real symplectic, and $\Sigma$ is positive diagonal.

DEFINITION 1.1. Let $J=\left[\begin{array}{cc}0 & I_{m} \\ -I_{m} & 0\end{array}\right]$.

1. A matrix $S \in \mathbb{R}^{2 m \times 2 m}$ is called symplectic if $S J S^{T}=J$.

2. A matrix $U \in \mathbb{R}^{2 m \times 2 m}$ is called orthogonal symplectic if $U^{T} U=I$ and $U J U^{T}=J$.

3. A matrix $A \in \mathbb{R}^{2 m \times 2 m}$ is called Hamiltonian if $J A=(J A)^{T}$.

The SVD-like decomposition (1.1) is closely related to the canonical forms of the real skew-symmetric matrix $B J B^{T}$ and the real Hamiltonian matrix $J B^{T} B$. By (1.1) and the symplectic property of $S$, we have the Schur-like form for $B J B^{T}$,

$$
B J B^{T}=Q\left[\begin{array}{cc|cc}
0 & 0 & \Sigma^{2} & 0 \\
0 & 0 & 0 & 0 \\
\hline-\Sigma^{2} & 0 & 0 & 0 \\
0 & 0 & 0 & 0
\end{array}\right] Q^{T},
$$

*Received by the editors June 22, 2002; accepted for publication (in revised form) by G. H. Golub May 30, 2004; published electronically May 6, 2005. This research was partially supported by NSF grant EPS-9874732, matching support from the state of Kansas, and the University of Kansas General Research Fund allocation 2301717.

http://www.siam.org/journals/simax/26-4/41052.html

${ }^{\dagger}$ Department of Mathematics, University of Kansas, Lawrence, KS 66045 (xu@math.ukans.edu). 
and the structured canonical form for $J B^{T} B$,

$$
J B^{T} B=S\left[\begin{array}{ccc|ccc}
0 & 0 & 0 & \Sigma^{2} & 0 & 0 \\
0 & 0 & 0 & 0 & 0 & 0 \\
0 & 0 & 0 & 0 & 0 & 0 \\
\hline-\Sigma^{2} & 0 & 0 & 0 & 0 & 0 \\
0 & -I & 0 & 0 & 0 & 0 \\
0 & 0 & 0 & 0 & 0 & 0
\end{array}\right] S^{-1}=: S \Gamma S^{-1} .
$$

(Note that the condensed matrix $\Gamma$ is still Hamiltonian.) In fact, let $\Sigma=\operatorname{diag}\left(\sigma_{1}, \ldots, \sigma_{p}\right)$. With appropriate permutations, (1.2) can be transformed to the real Schur form of $B J B^{T}$,

$$
\operatorname{diag}(\left[\begin{array}{cc}
0 & \sigma_{1}^{2} \\
-\sigma_{1}^{2} & 0
\end{array}\right], \ldots,\left[\begin{array}{cc}
0 & \sigma_{p}^{2} \\
-\sigma_{p}^{2} & 0
\end{array}\right], \underbrace{0, \ldots, 0}_{n-2 p})
$$

and (1.3) can be transformed to the real Jordan canonical form of $J B^{T} B$,

$$
\operatorname{diag}(\left[\begin{array}{cc}
0 & \sigma_{1}^{2} \\
-\sigma_{1}^{2} & 0
\end{array}\right], \ldots,\left[\begin{array}{cc}
0 & \sigma_{p}^{2} \\
-\sigma_{p}^{2} & 0
\end{array}\right], \underbrace{\left[\begin{array}{cc}
0 & 0 \\
-1 & 0
\end{array}\right], \ldots\left[\begin{array}{cc}
0 & 0 \\
-1 & 0
\end{array}\right]}_{q}, \underbrace{0, \ldots, 0}_{2(m-p-q)}) .
$$

In this paper we will develop a numerical method to compute the SVD-like decomposition (1.1). Our main goal is to use it to compute the structured canonical form (1.3) of the Hamiltonian matrices $J B^{T} B$.

The eigenvalue problem of such Hamiltonian matrices has a variety of applications. One example is the linear Hamiltonian system [19]

$$
\dot{x}(t)=J A x(t), \quad x(0)=x_{0},
$$

where $A \in \mathbb{R}^{2 m \times 2 m}$ is real symmetric positive definite. The solution of such a Hamiltonian system satisfies

$$
x^{T}(t) A x(t)=x_{0}^{T} A x_{0} \quad \forall t \geq 0 .
$$

This shows one fundamental principle of the Hamiltonian system, the conservation law. The solution $x(t)$ can be computed by using the structured canonical form of the Hamiltonian matrix $J A$. Since $A$ is positive definite, one can compute the factorization $A=B^{T} B$, say, the Cholesky factorization. After having computed the SVD-like decomposition of $B$, one has

$$
J A=S\left[\begin{array}{cc}
0 & \Sigma^{2} \\
-\Sigma^{2} & 0
\end{array}\right] S^{-1}=: S \Gamma S^{-1} .
$$

(Note that $\Gamma$ is slightly different from that in (1.3), because here $A$ is nonsingular.) The solution can be computed by the following formula:

$$
x(t)=S e^{\Gamma t} S^{-1} x_{0} .
$$

It is easily verified that for any $t, e^{\Gamma t}$ is symplectic. If $S$ is exactly symplectic, then 
one can verify that

$$
x^{T}(t) A x(t)=x^{T}(t) J^{-1}(J A) x(t)=\left(S e^{\Gamma t} S^{-1} x_{0}\right)^{T} J^{-1}\left(S \Gamma S^{-1}\right) S e^{\Gamma t} S^{-1} x_{0}=x_{0}^{T} A x_{0} .
$$

Numerically, for the solution $x(t)$ to obey the conservation law (1.4), one needs to compute the eigenvalues of $J A$ and the symplectic matrix $S$ accurately.

Another example involves the gyroscopic system $[8,13,17]$

$$
\ddot{q}+C \dot{q}+G q=0, \quad q(0)=q_{0}, \quad \dot{q}(0)=q_{1} .
$$

where $G \in \mathbb{R}^{m \times m}$ is symmetric and $C \in \mathbb{R}^{m \times m}$ is skew-symmetric. This system is related to the eigenvalue problem of the matrix

$$
F=\left[\begin{array}{cc}
-C & -G \\
I & 0
\end{array}\right]=\left[\begin{array}{cc}
-C & -I \\
I & 0
\end{array}\right]\left[\begin{array}{ll}
I & 0 \\
0 & G
\end{array}\right]
$$

When $G$ is positive semidefinite it has a full rank factorization $G=L L^{T}$. By using the equality

$$
\left[\begin{array}{cc}
-C & -I \\
I & 0
\end{array}\right]=\left[\begin{array}{cc}
-\frac{1}{2} C & I \\
I & 0
\end{array}\right] J\left[\begin{array}{cc}
\frac{1}{2} C & I \\
I & 0
\end{array}\right]
$$

$F$ is similar to the Hamiltonian matrix

$$
J\left[\begin{array}{cc}
\frac{1}{2} C & I \\
I & 0
\end{array}\right]\left[\begin{array}{cc}
I & 0 \\
0 & L L^{T}
\end{array}\right]\left[\begin{array}{cc}
-\frac{1}{2} C & I \\
I & 0
\end{array}\right]=J\left[\begin{array}{cc}
-\frac{1}{2} C & I \\
L^{T} & 0
\end{array}\right]^{T}\left[\begin{array}{cc}
-\frac{1}{2} C & I \\
L^{T} & 0
\end{array}\right] .
$$

Then the eigenvalue problem of $F$ can be solved by computing the SVD-like decomposition of $\left[\begin{array}{cc}-\frac{1}{2} C & I \\ L^{T} & 0\end{array}\right]$.

The eigenvalues of $J B^{T} B$ can be computed in many ways. For example, one can use the structure preserving method $[2,3]$. Since the eigenvalues of $J B^{T} B$ and $B J B^{T}$ are the same, a more efficient and reliable way is to use the QR method or the Jacobi method (e.g., $[15,11]$ ) to compute the eigenvalues of the skew-symmetric matrix $B J B^{T}$. A common problem of these methods is that they cannot compute the symplectic matrix $S$ simultaneously. Another problem is that the methods work on the explicit matrix product $J B^{T} B$ or $B J B^{T}$. The method that will be developed in this paper computes the SVD-like decomposition of $B$. So it computes both the eigenvalues of $J B^{T} B$ and the matrix $S$ simultaneously. Moreover, since it works only on the factor $B$, the eigenvalues of $J B^{T} B$ can be computed more accurately. This trick is not new; see, e.g., $[9,14]$. It has been also used to develop other singular value and eigenvalue methods $[5,12,1,10]$.

The basic idea of the method is introduced in section 2 , and the reduction and iteration processes are described in section 3. In these two sections we focus on a matrix $B$ with $B J B^{T}$ nonsingular. A detail reduction process for a general matrix $B$ is presented in section 4 . The first order error bound for the computed eigenvalues is provided in section 5. Numerical examples are given in section 6 . The conclusion is given in section 7 .

In this paper $\|\cdot\|$ denotes the spectral norm. 
2. The basic idea. We use the following procedure to compute an SVD-like decomposition. First compute a condensed form of $B$ by using only orthogonal transformations. Then use the condensed form to construct the SVD-like decomposition. The method for computing the condensed form is actually the implicit version of the QR-like method for the real skew-symmetric matrix $B J B^{T}$. In order to describe the method in a simple way, in this and the next sections for a matrix $B$ under consideration we assume that $B J B^{T}$ is nonsingular. With this assumption $B$ is necessarily of full row rank and has an even number of rows. A detailed process for a general matrix $B$ will be presented in section 4 .

For a nonsingular skew-symmetric matrix $K \in \mathbb{R}^{2 p \times 2 p}$ one can apply the QR-like algorithm to compute its Schur form. The algorithm consists of two steps. First apply a reduction procedure (see section 3 ) to $K$ to obtain a bidiagonal-like form

$$
Q_{1}^{T} K Q_{1}=\left[\begin{array}{cc}
0 & T \\
-T^{T} & 0
\end{array}\right]
$$

where $Q_{1}$ is real orthogonal and $T \in \mathbb{R}^{p \times p}$ is upper bidiagonal. Then apply the QR-like SVD iteration to $T$ to compute the SVD

$$
T=Z_{1} \Delta Z_{2}^{T}
$$

where $Z_{1}, Z_{2}$ are real orthogonal and $\Delta$ is positive diagonal. Let $Q=Q_{1}\left[\begin{array}{cc}Z_{1} & 0 \\ 0 & Z_{2}\end{array}\right]$. Then we have the Schur-like form

$$
Q^{T} K Q=\left[\begin{array}{cc}
0 & \Delta \\
-\Delta & 0
\end{array}\right]
$$

When $K=B J B^{T}$, we will develop an implicit version of the method by operating only on the factor $B$. Since $\left(Q^{T} B U\right) J\left(Q^{T} B U\right)^{T}=Q^{T}\left(B J B^{T}\right) Q$ for any orthogonal symplectic matrix $U$, we intend to determine an orthogonal matrix $Q$ and an orthogonal symplectic matrix $U$ such that $R=Q^{T} B U$ is block upper triangular and the product $R J R^{T}$ has the Schur-like form. Similarly we need two steps to compute such a decomposition. We first determine an orthogonal matrix $Q_{1}$ and an orthogonal symplectic matrix $U_{1}$ such that

$$
Q_{1}^{T} B U_{1}=\left[\begin{array}{cc}
B_{1} & B_{2} \\
0 & B_{3}
\end{array}\right]
$$

where $B_{1}, B_{2}, B_{3} \in \mathbb{R}^{p \times m}$, and

$$
Q_{1}^{T} B J B^{T} Q_{1}=\left[\begin{array}{cc}
B_{1} B_{2}^{T}-B_{2} B_{1}^{T} & B_{1} B_{3}^{T} \\
-B_{3} B_{1}^{T} & 0
\end{array}\right]=\left[\begin{array}{cc}
0 & B_{1} B_{3}^{T} \\
-B_{3} B_{1}^{T} & 0
\end{array}\right]
$$

has the bidiagonal-like form (2.1). (This implies that $B_{1} B_{2}^{T}=B_{2} B_{1}^{T}$ and $B_{1} B_{3}^{T}$ is upper bidiagonal.) We then apply an implicit version of the QR-like SVD iteration to $B_{1} B_{3}^{T}$, to obtain

$$
R_{1}=Z_{1}^{T} B_{1} W, \quad R_{3}=Z_{2}^{T} B_{3} W
$$

where $Z_{1}, Z_{2}, W$ are orthogonal and $R_{1} R_{3}^{T}=\Delta$ is positive diagonal. Let $Q=$ $Q_{1}\left[\begin{array}{cc}Z_{1} & 0 \\ 0 & Z_{2}\end{array}\right]$ and $U=U_{1}\left[\begin{array}{cc}W & 0 \\ 0 & W\end{array}\right]$ (which is orthogonal symplectic). Then

$$
R=Q^{T} B U=\left[\begin{array}{cc}
R_{1} & R_{2} \\
0 & R_{3}
\end{array}\right], \quad R_{2}=Z_{1}^{T} B_{2} W
$$


By (2.2) and (2.3), we have $Q^{T}\left(B J B^{T}\right) Q=R J R^{T}=\left[\begin{array}{cc}0 & \Delta \\ -\Delta & 0\end{array}\right]$.

The most condensed form that we can compute for $B$ is

$$
R=Q^{T} B U=\left[\begin{array}{cc|cc}
R_{11} & R_{12} & R_{13} & R_{14} \\
\hline 0 & 0 & R_{23} & 0
\end{array}\right]=:\left[\begin{array}{cc}
R_{1} & R_{2} \\
0 & R_{3}
\end{array}\right],
$$

where $R_{11}, R_{23} \in \mathbb{R}^{p \times p}, R_{11}$ is upper triangular, $R_{23}$ is lower triangular, and $R_{11} R_{23}^{T}=$ : $\Delta$ is positive diagonal. The detailed procedure will be presented in the next section. Let $\Delta=\operatorname{diag}\left(\delta_{1}, \ldots, \delta_{p}\right)$. After having obtained such a decomposition the eigenvalues of $B J B^{T}$ and $J B^{T} B$ are simply $\pm i \delta_{1}, \ldots, \pm i \delta_{p}$. Define $\Sigma=\sqrt{\Delta}$. The symplectic matrix $S$ in the SVD-like decomposition can be computed by the formula

$$
U\left[\begin{array}{cc|cc}
R_{23}^{T} \Sigma^{-1} & -\left(R_{23}^{T} \Sigma^{-1}\right)\left(R_{12}^{T} \Sigma^{-1}\right)^{T} & -R_{13}^{T} \Sigma^{-1} & -\left(R_{23}^{T} \Sigma^{-1}\right)\left(R_{14}^{T} \Sigma^{-1}\right)^{T} \\
0 & I & -R_{14}^{T} \Sigma^{-1} & 0 \\
\hline 0 & 0 & R_{11}^{T} \Sigma^{-1} & 0 \\
0 & 0 & R_{12}^{T} \Sigma^{-1} & I
\end{array}\right]
$$

and the SVD-like decomposition of $B$ is

$$
Q^{T} B S={ }_{p}^{p}\left(\begin{array}{cccc}
\Sigma & m-p & p & m-p \\
0 & 0 & 0 & 0 \\
0 & 0 & \Sigma & 0
\end{array}\right) .
$$

Note this is the decomposition only in the case that $B J B^{T}$ is nonsingular.

The method is summarized by the following algorithm.

Algorithm. Given a real matrix $B \in \mathbb{R}^{2 p \times 2 m}$ with $B J B^{T}$ nonsingular, the algorithm computes the eigenvalues of $J B^{T} B$ and $B J B^{T}$ or the SVD-like decomposition (2.6).

Step 1. Determine the orthogonal matrix $Q_{1}$ and the orthogonal symplectic matrix $U_{1}$ such that

$$
Q_{1}^{T} B U_{1}=\left[\begin{array}{cc|cc}
B_{11} & B_{12} & B_{13} & B_{14} \\
\hline 0 & 0 & B_{23} & 0
\end{array}\right]=:\left[\begin{array}{cc}
B_{1} & B_{2} \\
0 & B_{3}
\end{array}\right],
$$

where $B_{11}, B_{23} \in \mathbb{R}^{p \times p}, B_{11}$ is upper triangular, $B_{23}$ is lower triangular, $B_{11} B_{23}^{T}$ is upper bidiagonal, and $B_{1} B_{2}^{T}=B_{2} B_{1}^{T}$.

Step 2. Determine the orthogonal matrices $Z_{1}, Z_{2}, W$ such that

$$
R_{11}=Z_{1}^{T} B_{11} W, \quad R_{23}=Z_{2}^{T} B_{23} W,
$$

where $R_{11}$ is upper triangular, $R_{23}$ is lower triangular, and

$$
R_{11} R_{23}^{T}=\operatorname{diag}\left(\delta_{1}, \ldots, \delta_{p}\right)=: \Delta
$$

is positive diagonal.

Step 3. If only the eigenvalues of $J B^{T} B$ or $B J B^{T}$ are required, compute the nonzero eigenvalues $\pm i \delta_{1}, \ldots, \pm i \delta_{p}$ and stop. If the decomposition (2.6) is required, go to Step 4.

Step 4.

(a) Update $Q=Q_{1}\left[\begin{array}{cc}Z_{1} & 0 \\ 0 & Z_{2}\end{array}\right], U=U_{1} \operatorname{diag}(W, I, W, I)$, and

$$
R=\left[\begin{array}{cc|cc}
R_{11} & R_{12} & R_{13} & R_{14} \\
\hline 0 & 0 & R_{23} & 0
\end{array}\right],
$$

where $R_{12}=Z_{1}^{T} B_{12}, R_{13}=Z_{1}^{T} B_{13} W$, and $R_{14}=Z_{1}^{T} B_{14}$.

(b) Compute $\Sigma=\sqrt{\Delta}$.

(c) Use the formula (2.5) to compute $S$. 
3. Reduction and iteration. We need the following elementary matrices in our algorithm.

1. Set of Householder matrices:

$$
\mathcal{H}(\mathcal{I})=\left\{H=I_{n}-2 u u^{T} / u^{T} u \mid u \in \mathbb{R}^{n}, u_{j}=0, \forall j \notin \mathcal{I}\right\},
$$

where $\mathcal{I}$ is a subset of $\{1, \ldots, n\}$ giving the range of the columns and rows that $H$ operates on.

2. Set of Givens matrices:

$$
\mathcal{G}(i, j)=\left\{G \mid G=I_{n}-(1-\alpha)\left(e_{i} e_{i}^{T}+e_{j} e_{j}^{T}\right)+\beta\left(e_{i} e_{j}^{T}-e_{j} e_{i}^{T}\right), \alpha^{2}+\beta^{2}=1\right\} .
$$

3. Set of symplectic Householder matrices:

$$
\mathcal{H}^{s}(\mathcal{I})=\left\{H_{s} \mid H_{s}:=\left[\begin{array}{cc}
H & 0 \\
0 & H
\end{array}\right], H \in \mathcal{H}(\mathcal{I})\right\} .
$$

4. Sets of symplectic Givens matrices:
(a) $\mathcal{G}_{1}^{s}(k)=\left\{G_{s} \mid G_{s} \in \mathcal{G}(k, n+k) \subset \mathbb{R}^{2 n \times 2 n}\right\}$.
(b) $\mathcal{G}_{2}^{s}(i, j)=\left\{G_{s}=\left[\begin{array}{cc}G & 0 \\ 0 & G\end{array}\right] \mid G \in \mathcal{G}(i, j)\right\}$.
(c) $\mathcal{G}_{3}^{s}(i, j)=\left\{\begin{array}{l|l}G_{s} & \begin{array}{c}G_{s}=I_{2 n}-(1-\alpha)\left(e_{i} e_{i}^{T}+e_{j} e_{j}^{T}+e_{n+i} e_{n+i}^{T}+e_{n+j} e_{n+j}^{T}\right) \\ +\beta\left(e_{i} e_{n+j}^{T}+e_{j} e_{n+i}^{T}-e_{n+j} e_{i}^{T}-e_{n+i} e_{j}^{T}\right), \alpha^{2}+\beta^{2}=1\end{array}\end{array}\right\}$,

where $1 \leq i<j \leq n$.

5. Sets of symplectic permutations:

(a) $\mathcal{P}_{1}^{s}=\left\{\left[\begin{array}{cc}P & 0 \\ 0 & P\end{array}\right] \mid P\right.$ is a permutation $\}$.

(b) $\mathcal{P}_{2}^{s}(k)=\left\{P_{s} \mid P_{s}=I_{2 n}-\left(e_{k} e_{k}^{T}+e_{n+k} e_{n+k}^{T}\right)+\left(e_{k} e_{n+k}^{T}-e_{n+k} e_{k}^{T}\right)\right\}$.

In the algorithm Steps 3 and 4 are simple. So we consider only the implementations for Step 1 and 2 .

3.1. Implicit bidiagonal-like reduction. We use the following displays with a $6 \times 8$ matrix $B$ to illustrate the reduction process. In the displays, " 0 " and " $x$ " denote a zero and an arbitrary element, respectively. Note that our goal is to reduce $B$ to a condensed form (2.7) such that the explicit product $B J B^{T}$ has a bidiagonal-like form (2.1).

At the first stage we reduce the columns and rows 1 and 4 of $B J B^{T}$ implicitly. For this we first perform three orthogonal symplectic transformations $U_{1,1}, V_{1}, U_{1,2}$ successively, where $U_{1,1}, U_{1,2} \in \mathcal{H}^{s}(1: 4)$ and $V_{1} \in \mathcal{G}_{1}^{s}(1)$, on the columns of $B$ to annihilate $B(4,2: 4), B(4,1)$, and $B(4,6: 8):^{1}$

$$
\left[\begin{array}{llll|llll}
x & x & x & x & x & x & x & x \\
x & x & x & x & x & x & x & x \\
x & x & x & x & x & x & x & x \\
\hline 0 & 0 & 0 & 0 & x & 0 & 0 & 0 \\
x & x & x & x & x & x & x & x \\
x & x & x & x & x & x & x & x
\end{array}\right] .
$$

We then perform a Householder transformation $H_{1,1} \in \mathcal{H}(1: 3,5: 6)$ on the rows of

\footnotetext{
${ }^{1}$ Here we use the MATLAB forms to denote the entries, rows, and columns of a matrix.
} 
$B$ to annihilate $B(2: 3,1)$ and $B(5: 6,1)$ :

$$
\left[\begin{array}{llll|llll}
x & x & x & x & x & x & x & x \\
0 & x & x & x & x & x & x & x \\
0 & x & x & x & x & x & x & x \\
\hline 0 & 0 & 0 & 0 & x & 0 & 0 & 0 \\
0 & x & x & x & x & x & x & x \\
0 & x & x & x & x & x & x & x
\end{array}\right]
$$

Now the product $B\left(J B^{T}\right)$ has the form

$$
\left[\begin{array}{llll|llll}
x & x & x & x & x & x & x & x \\
0 & x & x & x & x & x & x & x \\
0 & x & x & x & x & x & x & x \\
\hline 0 & 0 & 0 & 0 & x & 0 & 0 & 0 \\
0 & x & x & x & x & x & x & x \\
0 & x & x & x & x & x & x & x
\end{array}\right]\left[\begin{array}{lll|lll}
x & x & x & x & x & x \\
x & x & x & 0 & x & x \\
x & x & x & 0 & x & x \\
x & x & x & 0 & x & x \\
\hline x & 0 & 0 & 0 & 0 & 0 \\
x & x & x & 0 & x & x \\
x & x & x & 0 & x & x \\
x & x & x & 0 & x & x
\end{array}\right]=\left[\begin{array}{lll|lll}
0 & x & x & x & x & x \\
x & 0 & x & 0 & x & x \\
x & x & 0 & 0 & x & x \\
\hline x & 0 & 0 & 0 & 0 & 0 \\
x & x & x & 0 & 0 & x \\
x & x & x & 0 & x & 0
\end{array}\right] .
$$

(Since $B J B^{T}$ is skew-symmetric, its diagonal elements are zero.) We still need to reduce the first column and row of $B J B^{T}$. For this we have to form the first column (but not the whole product) of $B J B^{T}$ explicitly, which has the pattern

$$
y_{1}=\left[\begin{array}{llllll}
0 & x & x & x & x & x
\end{array}\right]^{T} .
$$

Determine a Householder matrix $H_{1,2} \in \mathcal{H}(2: 3,5: 6)$ such that

$$
H_{1,2} y_{1}=\left[\begin{array}{llllll}
0 & 0 & 0 & x & x & 0
\end{array}\right]^{T} .
$$

Premultiply $B$ by $H_{1,2}$. Since $H_{1,2}$ does not work on rows 1 and 4 , it does not change the pattern of $B$. After this transformation

$$
B=\left[\begin{array}{llll|llll}
x & x & x & x & x & x & x & x \\
0 & x & x & x & x & x & x & x \\
0 & x & x & x & x & x & x & x \\
\hline 0 & 0 & 0 & 0 & x & 0 & 0 & 0 \\
0 & x & x & x & x & x & x & x \\
0 & x & x & x & x & x & x & x
\end{array}\right], \quad B J B^{T}=\left[\begin{array}{ccc|ccc}
0 & 0 & 0 & x & x & 0 \\
0 & 0 & x & 0 & x & x \\
0 & x & 0 & 0 & x & x \\
\hline x & 0 & 0 & 0 & 0 & 0 \\
x & x & x & 0 & 0 & x \\
0 & x & x & 0 & x & 0
\end{array}\right] .
$$

The second stage is similar. We reduce the columns and rows 2 and 5 of $B J B^{T}$. We first perform transformations $U_{2,1}, V_{2}, U_{2,2}$, where $U_{2,1}, U_{2,2} \in \mathcal{H}^{s}(2: 4)$ and $V_{2} \in \mathcal{G}_{1}^{s}(2)$, on the columns of $B$ to annihilate $B(5,3: 4), B(5,2)$, and $B(5,7: 8)$. Then perform a Householder transformation $H_{2,1} \in \mathcal{H}(2: 3,6)$ on the rows of $B$ to annihilate $B(3,2)$ and $B(6,2)$. Next we determine a Householder transformation $H_{2,2} \in \mathcal{H}(3,6)$ from the vector

$$
y_{2}=\left(B J B^{T}\right)(:, 2)=\left[\begin{array}{llllll}
0 & 0 & x & 0 & x & x
\end{array}\right]^{T},
$$

such that

$$
H_{2,2} y_{2}=\left[\begin{array}{llllll}
0 & 0 & 0 & 0 & x & x
\end{array}\right]^{T} .
$$


Premultiplying $B$ by $H_{2,2}$,

$$
B=\left[\begin{array}{llll|llll}
x & x & x & x & x & x & x & x \\
0 & x & x & x & x & x & x & x \\
0 & 0 & x & x & x & x & x & x \\
\hline 0 & 0 & 0 & 0 & x & 0 & 0 & 0 \\
0 & 0 & 0 & 0 & x & x & 0 & 0 \\
0 & 0 & x & x & x & x & x & x
\end{array}\right],
$$

and

$$
B J B^{T}=\left[\begin{array}{ccc|ccc}
0 & 0 & 0 & x & x & 0 \\
0 & 0 & 0 & 0 & x & x \\
0 & 0 & 0 & 0 & 0 & x \\
\hline x & 0 & 0 & 0 & 0 & 0 \\
x & x & 0 & 0 & 0 & 0 \\
0 & x & x & 0 & 0 & 0
\end{array}\right] .
$$

Now the product $B J B^{T}$ is in the bidiagonal-like form.

At the third stage we perform transformations $U_{3,1}, V_{3}, U_{3,2}$, where $U_{3,1}, U_{3,2} \in$ $\mathcal{H}^{s}(3: 4)$ and $V_{3} \in \mathcal{G}_{1}^{s}(3)$, on the columns of $B$ to annihilate $B(6,4), B(6,3)$, and $B(6,8)$ :

$$
B=\left[\begin{array}{lll|l|lll|l}
x & x & x & x & x & x & x & x \\
0 & x & x & x & x & x & x & x \\
0 & 0 & x & x & x & x & x & x \\
\hline 0 & 0 & 0 & 0 & x & 0 & 0 & 0 \\
0 & 0 & 0 & 0 & x & x & 0 & 0 \\
0 & 0 & 0 & 0 & x & x & x & 0
\end{array}\right] .
$$

We have got the form (2.7). Note that the symplectic transformations performed at the last stage do not change the bidiagonal-like form of $B J B^{T}$.

3.2. Implicit QR-like SVD iteration. We will give the implicit version of the implicit shift QR-like SVD iteration [11, sect. 8.6] on $B_{11} B_{23}^{T}$. For a technical reason related to decoupling and deflation, before iteration we transform $B_{23}$ to a lower Hessenberg form such that $B_{11} B_{23}^{T}$ is lower bidiagonal. The transformations can be performed as follows. For $j=1, \ldots, p-1$, we construct a sequence of Givens matrices $G_{j} \in \mathcal{G}(j, j+1)$ such that $\left(B_{11} B_{23}^{T}\right) G_{1} \cdots G_{p-1}$ becomes lower bidiagonal. (To construct $G_{j}$ we need to compute $\left(B_{11} B_{23}^{T}\right)(j, j: j+1)$.) Update $B_{23}:=G_{p-1}^{T} \cdots G_{1}^{T} B_{23}$. Then $B_{23}$ becomes lower Hessenberg. $B_{11}$ is still upper triangular, but now $B_{11} B_{23}^{T}$ is lower bidiagonal.

When some diagonal or subdiagonal elements of $B_{11} B_{23}^{T}$ are zero we can decouple it into smaller unreduced lower bidiagonal blocks. With the assumption that $B J B^{T}$ is nonsingular all diagonal elements of $B_{11} B_{23}^{T}$ are nonzero. This is obvious from the factorization $B J B^{T}=Q_{1}\left[\begin{array}{cc}0 & B_{11} B_{23}^{T} B_{11} \\ 0\end{array}\right] Q_{1}^{T}$. Moreover, $B_{11}$ is nonsingular. Hence its diagonal elements are nonzero. The $j$ th subdiagonal element of $B_{11} B_{23}^{T}$ has the form $^{2} B_{11}(j+1, j+1) B_{23}(j, j+1)$. Because $B_{11}(j+1, j+1) \neq 0$, the $j$ th subdiagonal

\footnotetext{
${ }^{2}$ This is why we transform $B_{23}$ to a lower Hessenberg form. In the upper bidiagonal case the $j$ th superdiagonal element of $B_{11} B_{23}^{T}$ is in a dot product form $B_{11}(j, j) B_{23}(j+1, j)+B_{11}(j, j+$ 1) $B_{23}(j+1, j+1)$. It may happen that this dot product is small but all four elements are not small. When this happens, we have the difficulty of doing the decoupling or deflation.
} 
element of $B_{11} B_{23}^{T}$ is zero if and only if the $j$ th superdiagonal element of $B_{23}$ is zero. With this observation, in practice when some superdiagonal elements of $B_{23}$ are zero or suitably small we set them to be zero and decouple $B_{11} B_{23}^{T}$ into smaller unreduced lower bidiagonal blocks. We then perform the following implicit version of the QR-like SVD iterations to each pair of small diagonal blocks from $B_{11}$ and $B_{23}$ corresponding to each unreduced block in $B_{11} B_{23}^{T}$ to compute (2.8). The criterion for decoupling or deflation that we use is

$$
\left|B_{23}(j, j+1)\right| \leq \varepsilon\left(\left|B_{23}(j, j)\right|+\left|B_{23}(j+1, j)\right|+\left|B_{23}(j+1, j+1)\right|\right),
$$

where $\varepsilon$ is the machine precision. With this criterion decoupling or deflation will cause an error in $B$ of order $\varepsilon\|B\|$.

We use the matrices $B_{11}, B_{23}$ with size $4 \times 4$ to illustrate one step of iteration. Initially $B_{11}$ is upper triangular, $B_{23}$ is lower Hessenberg, and $B_{11} B_{23}^{T}$ is lower bidiagonal. Without loss of generality we assume that $B_{11} B_{23}^{T}$ is unreduced. Let $\delta>0$ be a shift. ${ }^{3}$ Let $A$ be the leading $2 \times 2$ principal submatrix of $B_{11} B_{23}^{T} B_{23} B_{11}^{T}$. We first determine a Givens matrix $G_{1} \in \mathcal{G}(1,2)$, in which the leading $2 \times 2$ principal submatrix is a Givens rotation that transforms $A-\delta I$ to an upper triangular form. Perform $G_{1}$ on the rows of $B_{11}$ :

$$
B_{11}=\left[\begin{array}{llll}
x & x & x & x \\
\otimes & x & x & x \\
0 & 0 & x & x \\
0 & 0 & 0 & x
\end{array}\right]
$$

where " $\otimes$ " denotes an unwanted nonzero element. Now the product becomes

$$
B_{11} B_{23}^{T}=\left[\begin{array}{cccc}
x & \otimes & 0 & 0 \\
x & x & 0 & 0 \\
0 & x & x & 0 \\
0 & 0 & x & x
\end{array}\right] .
$$

Perform a Givens transformation $W_{1} \in \mathcal{G}(1,2)$ on the columns of $B_{11}$ to annihilate $B_{11}(2,1)$ and perform it also on the columns of $B_{23}$ :

$$
B_{11}=\left[\begin{array}{llll}
x & x & x & x \\
0 & x & x & x \\
0 & 0 & x & x \\
0 & 0 & 0 & x
\end{array}\right], \quad B_{23}=\left[\begin{array}{llll}
x & x & 0 & 0 \\
x & x & x & 0 \\
x & x & x & x \\
x & x & x & x
\end{array}\right] .
$$

This transformation does not change the pattern of $B_{11} B_{23}^{T}$. Next we determine a Givens matrix $S_{1} \in \mathcal{G}(1,2)$ to annihilate $\left(B_{11} B_{23}^{T}\right)(1,2)$. (Again, in order to construct $S_{1}$ we need to compute $\left(B_{11} B_{23}^{T}\right)(1,1: 2)$.) Perform $S_{1}$ on the rows of $B_{23}$ :

$$
B_{23}=\left[\begin{array}{llll}
x & x & \otimes & 0 \\
x & x & x & 0 \\
x & x & x & x \\
x & x & x & x
\end{array}\right],
$$

\footnotetext{
${ }^{3}$ We actually use the Wilkinson shift, one of the eigenvalues of the tailing $2 \times 2$ principal submatrix of $B_{11} B_{23}^{T} B_{23} B_{11}^{T}$.
} 
and now

$$
B_{11} B_{23}^{T}=\left[\begin{array}{cccc}
x & 0 & 0 & 0 \\
x & x & 0 & 0 \\
\otimes & x & x & 0 \\
0 & 0 & x & x
\end{array}\right] .
$$

To annihilate $\left(B_{11} B_{23}^{T}\right)(3,1)$ we first perform a Givens transformation $W_{2} \in \mathcal{G}(2,3)$ on the columns of $B_{23}$ to annihilate $B_{23}(1,3)$. Perform $W_{2}$ also on the columns of $B_{11}$ :

$$
B_{11}=\left[\begin{array}{llll}
x & x & x & x \\
0 & x & x & x \\
0 & \otimes & x & x \\
0 & 0 & 0 & x
\end{array}\right], \quad B_{23}=\left[\begin{array}{llll}
x & x & 0 & 0 \\
x & x & x & 0 \\
x & x & x & x \\
x & x & x & x
\end{array}\right] .
$$

Then we perform a Givens transformation $G_{2} \in \mathcal{G}(2,3)$ on the rows of $B_{11}$ to annihilate $B_{11}(3,2)$ :

$$
B_{11}=\left[\begin{array}{cccc}
x & x & x & x \\
0 & x & x & x \\
0 & 0 & x & x \\
0 & 0 & 0 & x
\end{array}\right]
$$

At this stage

$$
B_{11} B_{23}^{T}=\left[\begin{array}{cccc}
x & 0 & 0 & 0 \\
x & x & \otimes & 0 \\
0 & x & x & 0 \\
0 & 0 & x & x
\end{array}\right]
$$

So $\left(B_{11} B_{23}^{T}\right)(3,1)$ has been annihilated and the bulge has been chased to the $(2,3)$ place. In a similar way we can chase the bulge down-rightwards until it disappears. The rest of the reductions are illustrated by the following displays, where $B_{11}$ and $B_{23}$ are displayed simultaneously, the Givens transformation $G_{j} \in \mathcal{G}(j, j+1)$ operates only on the rows of $B_{11}, S_{j} \in \mathcal{G}(j, j+1)$ operates only on the rows of $B_{23}$, and $W_{j} \in \mathcal{G}(j, j+1)$ operates on the columns of both $B_{11}$ and $B_{23}$.

$$
\begin{aligned}
& {\left[\begin{array}{llll}
x & x & x & x \\
0 & x & x & x \\
0 & 0 & x & x \\
0 & 0 & 0 & x
\end{array}\right], \quad\left[\begin{array}{llll}
x & x & 0 & 0 \\
x & x & x & 0 \\
x & x & x & x \\
x & x & x & x
\end{array}\right] \stackrel{S_{2}}{\longrightarrow}\left[\begin{array}{llll}
x & x & x & x \\
0 & x & x & x \\
0 & 0 & x & x \\
0 & 0 & 0 & x
\end{array}\right], \quad\left[\begin{array}{llll}
x & x & 0 & 0 \\
x & x & x & \otimes \\
x & x & x & x \\
x & x & x & x
\end{array}\right]} \\
& \stackrel{W_{3}}{\longrightarrow}\left[\begin{array}{cccc}
x & x & x & x \\
0 & x & x & x \\
0 & 0 & x & x \\
0 & 0 & \otimes & x
\end{array}\right], \quad\left[\begin{array}{cccc}
x & x & 0 & 0 \\
x & x & x & 0 \\
x & x & x & x \\
x & x & x & x
\end{array}\right] \stackrel{G_{3}}{\longrightarrow}\left[\begin{array}{cccc}
x & x & x & x \\
0 & x & x & x \\
0 & 0 & x & x \\
0 & 0 & 0 & x
\end{array}\right],\left[\begin{array}{cccc}
x & x & 0 & 0 \\
x & x & x & 0 \\
x & x & x & x \\
x & x & x & x
\end{array}\right] \\
& \stackrel{S_{3}}{\longrightarrow}\left[\begin{array}{llll}
x & x & x & x \\
0 & x & x & x \\
0 & 0 & x & x \\
0 & 0 & 0 & x
\end{array}\right],\left[\begin{array}{llll}
x & x & 0 & 0 \\
x & x & x & 0 \\
x & x & x & x \\
x & x & x & x
\end{array}\right] \text {. }
\end{aligned}
$$

We have finished one step of iteration. 
We now check the superdiagonal elements of $B_{23}$. If some of them satisfy (3.1), we replace them by zero and decouple or deflate $B_{11} B_{23}^{T}$. We then run another step of iteration on $B_{11}$ and $B_{23}$ or a pair of diagonal blocks from them. Repeat the iterations and finally $B_{23}$ becomes lower triangular and we have (2.8).

The algorithm costs about two to three times as much as the QR-like algorithm applied to the explicit product $B J B^{T}$.

4. General case. For a general matrix $B \in \mathbb{R}^{n \times 2 m}$ additional work needs to be done. If $\operatorname{rank} B<n$, initially we need to compute a factorization

$$
B=Q_{0}\left[\begin{array}{c}
B_{0} \\
0
\end{array}\right]
$$

where $Q_{0}$ is orthogonal and $B_{0}$ is of full row rank. This can be done by the QR factorization with the column pivoting method (see [6]), the rank-revealing QR (see [7]), or the SVD algorithm (see [11, sect. 8.6]).

Next we apply the reduction process to $B_{0}$. But now we have to modify the above reduction process slightly. The reason is that even if $B_{0}$ is of full row rank, the product $B_{0} J B_{0}^{T}$ may be singular. In this case at certain stages of reductions some diagonal elements of block $B_{11}$ or $B_{23}$ will be zero and we need to deflate the zero eigenvalues of $B_{0} J B_{0}^{T}$. Because of this, we have to reduce matrix $B_{0}$ to a more generalized condensed form

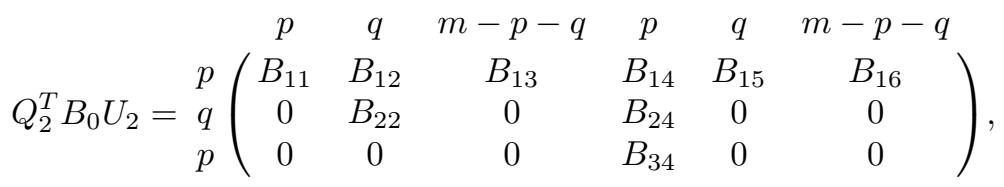

where $Q_{2}$ is orthogonal, $U_{2}$ is orthogonal symplectic, $B_{11}, B_{22}$ are nonsingular and upper triangular, $B_{34}$ is nonsingular and lower triangular, $B_{11} B_{34}^{T}$ is upper bidiagonal, and

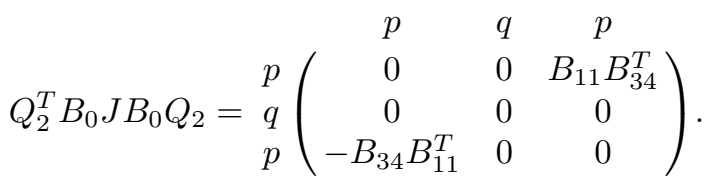

The reduction procedure will be illustrated below. We then apply the same iteration procedure described in subsection 3.2 to $B_{11}, B_{34}$ to compute

$$
R_{11}=Z_{1}^{T} B_{11} W, \quad R_{34}=Z_{2}^{T} B_{34} W,
$$

where $Z_{1}, Z_{2}, W$ are orthogonal, $R_{11}$ is upper triangular, $R_{34}$ is lower triangular, and $\Delta:=R_{11} R_{34}^{T}$ is positive diagonal. Similarly, combining them with (4.2) and (4.1) we can determine the orthogonal matrix $Q$ and the orthogonal symplectic matrix $U$ to obtain the generalized version of (2.4),

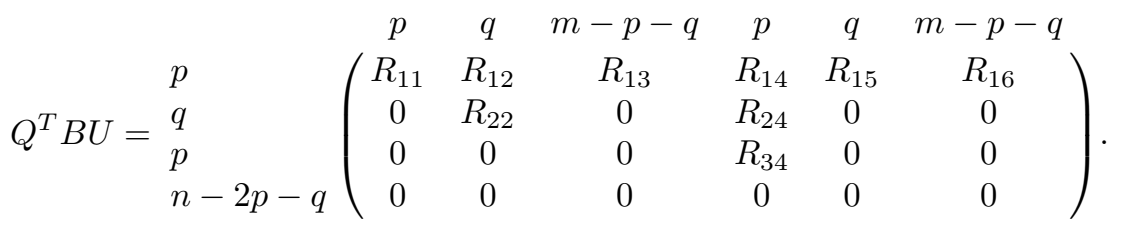


Let $\Sigma=\sqrt{\Delta}$. The symplectic matrix $S$ can be computed by the formula

$U\left[\begin{array}{ccc|ccc}X & -X\left(R_{12}^{T} \Sigma^{-1}\right)^{T} & -X\left(R_{13}^{T} \Sigma^{-1}\right)^{T} & -R_{14}^{T} \Sigma^{-1} & -X\left(R_{15}^{T} \Sigma^{-1}\right)^{T} & -X\left(R_{16}^{T} \Sigma^{-1}\right)^{T} \\ 0 & I & 0 & -R_{15}^{T} \Sigma^{-1} & 0 & 0 \\ 0 & 0 & I & -R_{16}^{T} \Sigma^{-1} & 0 & 0 \\ \hline 0 & 0 & 0 & R_{11}^{T} \Sigma^{-1} & 0 & 0 \\ 0 & 0 & 0 & R_{12}^{T} \Sigma^{-1} & I & 0 \\ 0 & 0 & 0 & R_{13}^{T} \Sigma^{-1} & 0 & I\end{array}\right]$,

where $X=R_{34}^{T} \Sigma^{-1}$. Finally we have the SVD-like decomposition

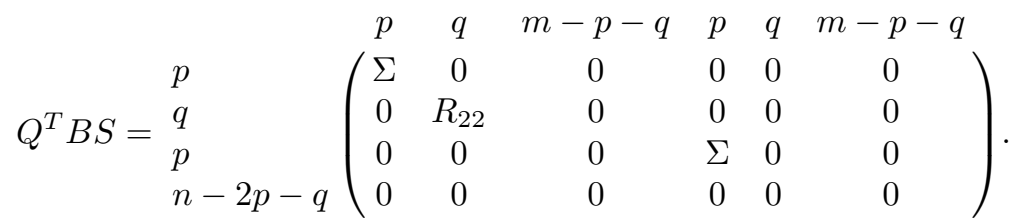

(If necessary one can multiply the symplectic matrix $\operatorname{diag}\left(I, R_{22}^{-1}, I ; I, R_{22}^{T}, I\right)$ from the right to replace $R_{22}$ by $I$.)

In the following we will show the reduction procedure for computing the condensed form (4.2). The procedure consists of two steps. In step 1 we will reduce $B_{0}$ to

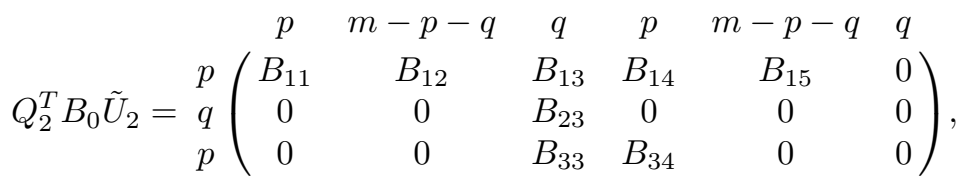

where $Q_{2}$ is orthogonal, $\tilde{U}_{2}$ is orthogonal symplectic, $B_{11}, B_{23}$ are nonsingular and upper triangular, and $B_{34}$ is nonsingular and lower triangular, such that $Q_{2}^{T}\left(B_{0} J B_{0}^{T}\right) Q_{2}$ has the bidiagonal-like form (4.3). In step 2 we will perform only orthogonal symplectic transformations on the columns to transform (4.4) to (4.2). Note that step 2 does not change the bidiagonal-like form of $Q_{2}^{T}\left(B_{0} J B_{0}^{T}\right) Q_{2}$.

Let us describe step 1 in an inductive way. Suppose that at a certain stage we have reduced $B_{0}$ to

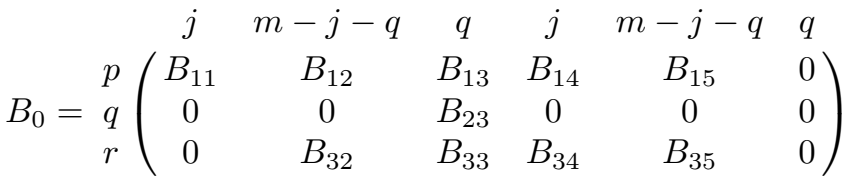

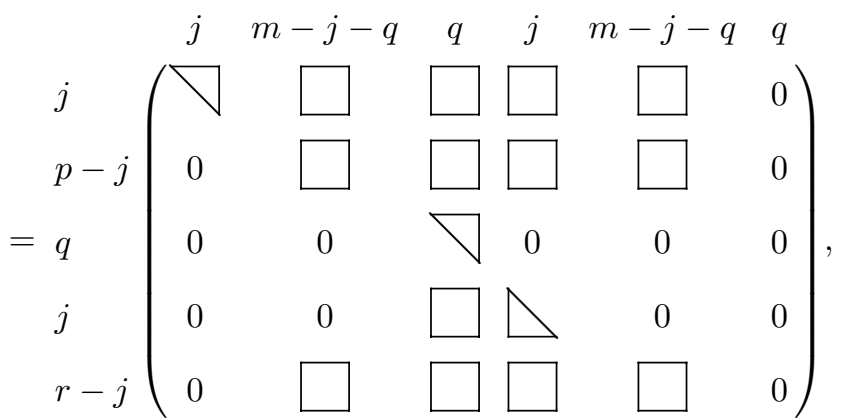


and

$$
B_{0} J B_{0}=\begin{aligned}
& j \\
& j-j \\
& j \\
& j-j \\
& \hdashline
\end{aligned}\left(\begin{array}{ccccc}
0 & 0 & 0 & \searrow & * \\
0 & \square & 0 & 0 & \square \\
0 & 0 & 0 & 0 & 0 \\
* & 0 & 0 & 0 & 0 \\
& \square & 0 & 0 & \square
\end{array}\right),
$$

where $r=p$ or $p+1, j \leq p$. (Initially we partition $B_{0}$ to the block form with $j=q=0$, and $r=p$ if $B_{0}$ has $2 p$ (even) rows or $r=p+1$ if $B_{0}$ has $2 p+1$ (odd) rows.) Note that in (4.5) when $j=p=r$ it is just (4.4) and we are done.

If $j \leq p$, we continue the reduction process. We first perform orthogonal symplectic transformations on the columns of $B_{0}$ to annihilate $B_{0}(p+q+j+1, j+1: m-q)$ and $B_{0}(p+q+j+1, m+j+2: 2 m-q)$, and then perform a Householder transformation on the rows of $B_{0}$ to annihilate $B_{0}(j+2: p, j+1)$ and $B_{0}(p+q+j+2: p+q+r, j+1)$. After this step we have two cases.

(a) $B_{0}(j+1, j+1) \neq 0$. We determine another Householder matrix to annihilate the elements from $j+2$ to $p$ and from $p+q+j+3$ to $p+q+r$ on the $(j+1)$ th column/row of $B_{0} J B_{0}^{T}$. Premultiply $B_{0}$ by this Householder matrix. Then $B_{0}$ and $B_{0} J B_{0}^{T}$ again have the block forms (4.5) and (4.6), respectively, but $j:=j+1$. We have done one step of regular reduction as in subsection 3.1.

(b) $B_{0}(j+1, j+1)=0$. We need to deflate the zero eigenvalue of $B_{0} J B_{0}^{T}$. We have two subcases:

$$
\begin{aligned}
& \text { (b1) } r=p+1 \text { and } \\
& \text { (b2) } r=p .
\end{aligned}
$$

For the first subcase the deflation is illustrated by a matrix with $j=2, p=4, r=5$, $q=2$, and $m=8$ :

$$
B_{0}=\left[\begin{array}{ll|llll|ll|ll|llll|ll}
x & x & x & x & x & x & x & x & x & x & x & x & x & x & 0 & 0 \\
0 & x & x & x & x & x & x & x & x & x & x & x & x & x & 0 & 0 \\
0 & 0 & 0 & x & x & x & x & x & x & x & x & x & x & x & 0 & 0 \\
0 & 0 & 0 & x & x & x & x & x & x & x & x & x & x & x & 0 & 0 \\
\hline 0 & 0 & 0 & 0 & 0 & 0 & x & x & 0 & 0 & 0 & 0 & 0 & 0 & 0 & 0 \\
0 & 0 & 0 & 0 & 0 & 0 & 0 & x & 0 & 0 & 0 & 0 & 0 & 0 & 0 & 0 \\
\hline 0 & 0 & 0 & 0 & 0 & 0 & x & x & x & 0 & 0 & 0 & 0 & 0 & 0 & 0 \\
0 & 0 & 0 & 0 & 0 & 0 & x & x & x & x & 0 & 0 & 0 & 0 & 0 & 0 \\
0 & 0 & 0 & 0 & 0 & 0 & x & x & x & x & x & 0 & 0 & 0 & 0 & 0 \\
0 & 0 & 0 & x & x & x & x & x & x & x & x & x & x & x & 0 & 0 \\
0 & 0 & 0 & x & x & x & x & x & x & x & x & x & x & x & 0 & 0
\end{array}\right]
$$


and

$$
B_{0} J B_{0}^{T}=\left[\begin{array}{cccc|cc|ccccc}
0 & 0 & 0 & 0 & 0 & 0 & x & x & 0 & 0 & 0 \\
0 & 0 & 0 & 0 & 0 & 0 & 0 & x & x & 0 & 0 \\
0 & 0 & 0 & x & 0 & 0 & 0 & 0 & 0 & x & x \\
0 & 0 & x & 0 & 0 & 0 & 0 & 0 & 0 & x & x \\
\hline 0 & 0 & 0 & 0 & 0 & 0 & 0 & 0 & 0 & 0 & 0 \\
0 & 0 & 0 & 0 & 0 & 0 & 0 & 0 & 0 & 0 & 0 \\
\hline x & 0 & 0 & 0 & 0 & 0 & 0 & 0 & 0 & 0 & 0 \\
x & x & 0 & 0 & 0 & 0 & 0 & 0 & 0 & 0 & 0 \\
0 & x & 0 & 0 & 0 & 0 & 0 & 0 & 0 & 0 & 0 \\
0 & 0 & x & x & 0 & 0 & 0 & 0 & 0 & 0 & x \\
0 & 0 & x & x & 0 & 0 & 0 & 0 & 0 & x & 0
\end{array}\right]
$$

For the explicit product we can perform a sequence of Givens transformations $G_{1} \in$ $\mathcal{G}(8,9)$ and $G_{2} \in \mathcal{G}(7,8)$ on both the columns and rows to annihilate $\left(B_{0} J B_{0}^{T}\right)(2,8)$, $\left(B_{0} J B_{0}^{T}\right)(1,7)$ and $\left(B_{0} J B_{0}^{T}\right)(8,2),\left(B_{0} J B_{0}^{T}\right)(7,1)$. With repartitioning we again have the form (4.6) but with $q=3$ :

$$
B_{0} J B_{0}^{T}=\left[\begin{array}{cccc|ccc|cccc}
0 & 0 & 0 & 0 & 0 & 0 & 0 & x & x & 0 & 0 \\
0 & 0 & 0 & 0 & 0 & 0 & 0 & 0 & x & 0 & 0 \\
0 & 0 & 0 & x & 0 & 0 & 0 & 0 & 0 & x & x \\
0 & 0 & x & 0 & 0 & 0 & 0 & 0 & 0 & x & x \\
\hline 0 & 0 & 0 & 0 & 0 & 0 & 0 & 0 & 0 & 0 & 0 \\
0 & 0 & 0 & 0 & 0 & 0 & 0 & 0 & 0 & 0 & 0 \\
0 & 0 & 0 & 0 & 0 & 0 & 0 & 0 & 0 & 0 & 0 \\
\hline x & 0 & 0 & 0 & 0 & 0 & 0 & 0 & 0 & 0 & 0 \\
x & x & 0 & 0 & 0 & 0 & 0 & 0 & 0 & 0 & 0 \\
0 & 0 & x & x & 0 & 0 & 0 & 0 & 0 & 0 & x \\
0 & 0 & x & x & 0 & 0 & 0 & 0 & 0 & x & 0
\end{array}\right]
$$

The corresponding implicit version is as follows. We first perform a sequence of the symplectic Givens transformations $U_{1} \in \mathcal{G}_{2}^{s}(2,3), U_{2} \in \mathcal{G}_{2}^{s}(1,2)$ on the columns of $B_{0}$ to annihilate $B_{0}(2,2)$ and $B_{0}(1,1)$ :

$$
B_{0}=\left[\begin{array}{ll|llll|ll|ll|llll|ll}
0 & x & x & x & x & x & x & x & x & x & x & x & x & x & 0 & 0 \\
0 & 0 & x & x & x & x & x & x & x & x & x & x & x & x & 0 & 0 \\
0 & 0 & 0 & x & x & x & x & x & x & x & x & x & x & x & 0 & 0 \\
0 & 0 & 0 & x & x & x & x & x & x & x & x & x & x & x & 0 & 0 \\
\hline 0 & 0 & 0 & 0 & 0 & 0 & x & x & 0 & 0 & 0 & 0 & 0 & 0 & 0 & 0 \\
0 & 0 & 0 & 0 & 0 & 0 & 0 & x & 0 & 0 & 0 & 0 & 0 & 0 & 0 & 0 \\
\hline 0 & 0 & 0 & 0 & 0 & 0 & x & x & x & \otimes & 0 & 0 & 0 & 0 & 0 & 0 \\
0 & 0 & 0 & 0 & 0 & 0 & x & x & x & x & \otimes & 0 & 0 & 0 & 0 & 0 \\
0 & 0 & 0 & 0 & 0 & 0 & x & x & x & x & x & 0 & 0 & 0 & 0 & 0 \\
0 & 0 & 0 & x & x & x & x & x & x & x & x & x & x & x & 0 & 0 \\
0 & 0 & 0 & x & x & x & x & x & x & x & x & x & x & x & 0 & 0
\end{array}\right] .
$$

Then perform Givens transformations $G_{1} \in \mathcal{G}(8,9)$ and $G_{2} \in \mathcal{G}(7,8)$ on the rows of 
$B_{0}$ to annihilate the unwanted elements $B_{0}(8,11)$ and $B_{0}(7,10)$ :

$$
B_{0}=\left[\begin{array}{ll|llll|ll|ll|llll|ll}
0 & x & x & x & x & x & x & x & x & x & x & x & x & x & 0 & 0 \\
0 & 0 & x & x & x & x & x & x & x & x & x & x & x & x & 0 & 0 \\
0 & 0 & 0 & x & x & x & x & x & x & x & x & x & x & x & 0 & 0 \\
0 & 0 & 0 & x & x & x & x & x & x & x & x & x & x & x & 0 & 0 \\
\hline 0 & 0 & 0 & 0 & 0 & 0 & x & x & 0 & 0 & 0 & 0 & 0 & 0 & 0 & 0 \\
0 & 0 & 0 & 0 & 0 & 0 & 0 & x & 0 & 0 & 0 & 0 & 0 & 0 & 0 & 0 \\
\hline 0 & 0 & 0 & 0 & 0 & 0 & x & x & x & 0 & 0 & 0 & 0 & 0 & 0 & 0 \\
0 & 0 & 0 & 0 & 0 & 0 & x & x & x & x & 0 & 0 & 0 & 0 & 0 & 0 \\
0 & 0 & 0 & 0 & 0 & 0 & x & x & x & x & x & 0 & 0 & 0 & 0 & 0 \\
0 & 0 & 0 & x & x & x & x & x & x & x & x & x & x & x & 0 & 0 \\
0 & 0 & 0 & x & x & x & x & x & x & x & x & x & x & x & 0 & 0
\end{array}\right] .
$$

Now by using the pattern of $B_{0}$ one can see that $B_{0} J B_{0}^{T}$ has the form (4.7). To transform $B_{0}$ back to the block form (4.5) next we perform a symplectic permutation $P_{1} \in \mathcal{P}_{1}^{s}$ to move the columns 1 and 9 of $B_{0}$ to columns 6 and 14 , respectively. Then we perform a symplectic permutation $P_{2} \in \mathcal{P}_{2}^{s}(6)$ to interchange the columns 6 and 14 . With repartitioning,

$$
B_{0}=\left[\begin{array}{ll|lll|lll|ll|lll|lll}
x & x & x & x & x & x & x & x & x & x & x & x & x & 0 & 0 & 0 \\
0 & x & x & x & x & x & x & x & x & x & x & x & x & 0 & 0 & 0 \\
0 & 0 & x & x & x & x & x & x & x & x & x & x & x & 0 & 0 & 0 \\
0 & 0 & x & x & x & x & x & x & x & x & x & x & x & 0 & 0 & 0 \\
\hline 0 & 0 & 0 & 0 & 0 & 0 & x & x & 0 & 0 & 0 & 0 & 0 & 0 & 0 & 0 \\
0 & 0 & 0 & 0 & 0 & 0 & 0 & x & 0 & 0 & 0 & 0 & 0 & 0 & 0 & 0 \\
0 & 0 & 0 & 0 & 0 & x & x & x & 0 & 0 & 0 & 0 & 0 & 0 & 0 & 0 \\
\hline 0 & 0 & 0 & 0 & 0 & x & x & x & x & 0 & 0 & 0 & 0 & 0 & 0 & 0 \\
0 & 0 & 0 & 0 & 0 & x & x & x & x & x & 0 & 0 & 0 & 0 & 0 & 0 \\
0 & 0 & x & x & x & x & x & x & x & x & x & x & x & 0 & 0 & 0 \\
0 & 0 & x & x & x & x & x & x & x & x & x & x & x & 0 & 0 & 0
\end{array}\right] .
$$

Note that these permutations do not change the form of $B_{0} J B_{0}^{T}$. To maintain the block $B_{23}$ in upper triangular form we perform a row permutation to move row 7 to row 5:

$$
B_{0}=\left[\begin{array}{ll|lll|lll|ll|llll|ll}
x & x & x & x & x & x & x & x & x & x & x & x & x & 0 & 0 & 0 \\
0 & x & x & x & x & x & x & x & x & x & x & x & x & 0 & 0 & 0 \\
0 & 0 & x & x & x & x & x & x & x & x & x & x & x & 0 & 0 & 0 \\
0 & 0 & x & x & x & x & x & x & x & x & x & x & x & 0 & 0 & 0 \\
\hline 0 & 0 & 0 & 0 & 0 & x & x & x & 0 & 0 & 0 & 0 & 0 & 0 & 0 & 0 \\
0 & 0 & 0 & 0 & 0 & 0 & x & x & 0 & 0 & 0 & 0 & 0 & 0 & 0 & 0 \\
0 & 0 & 0 & 0 & 0 & 0 & 0 & x & 0 & 0 & 0 & 0 & 0 & 0 & 0 & 0 \\
\hline 0 & 0 & 0 & 0 & 0 & x & x & x & x & 0 & 0 & 0 & 0 & 0 & 0 & 0 \\
0 & 0 & 0 & 0 & 0 & x & x & x & x & x & 0 & 0 & 0 & 0 & 0 & 0 \\
0 & 0 & x & x & x & x & x & x & x & x & x & x & x & 0 & 0 & 0 \\
0 & 0 & x & x & x & x & x & x & x & x & x & x & x & 0 & 0 & 0
\end{array}\right] .
$$

Then $B_{0}$ and $B_{0} J B_{0}^{T}$ again have the forms (4.5) and (4.6), respectively, but now $r:=r-1$ and $q:=q+1$. 
For the second subcase the reduction procedure is illustrated by a matrix with $j=2, p=5, r=5, q=1$, and $m=8$ :

$$
B_{0}=\left[\begin{array}{ll|lllll|l|ll|lllll|l}
x & x & x & x & x & x & x & x & x & x & x & x & x & x & x & 0 \\
0 & x & x & x & x & x & x & x & x & x & x & x & x & x & x & 0 \\
0 & 0 & \mathbf{0} & x & x & x & x & x & x & x & x & x & x & x & x & 0 \\
0 & 0 & 0 & x & x & x & x & x & x & x & x & x & x & x & x & 0 \\
0 & 0 & 0 & x & x & x & x & x & x & x & x & x & x & x & x & 0 \\
\hline 0 & 0 & 0 & 0 & 0 & 0 & 0 & x & 0 & 0 & 0 & 0 & 0 & 0 & 0 & 0 \\
\hline 0 & 0 & 0 & 0 & 0 & 0 & x & x & x & 0 & 0 & 0 & 0 & 0 & 0 & 0 \\
0 & 0 & 0 & 0 & 0 & 0 & x & x & x & x & 0 & 0 & 0 & 0 & 0 & 0 \\
0 & 0 & 0 & 0 & 0 & 0 & x & x & x & x & x & 0 & 0 & 0 & 0 & 0 \\
0 & 0 & 0 & x & x & x & x & x & x & x & x & x & x & x & x & 0 \\
0 & 0 & 0 & x & x & x & x & x & x & x & x & x & x & x & x & 0
\end{array}\right],
$$

and

$$
B_{0} J B_{0}^{T}=\left[\begin{array}{ccccc|c|ccccc}
0 & 0 & 0 & 0 & 0 & 0 & x & x & 0 & 0 & 0 \\
0 & 0 & 0 & 0 & 0 & 0 & 0 & x & x & 0 & 0 \\
0 & 0 & 0 & x & x & 0 & 0 & 0 & 0 & x & x \\
0 & 0 & x & 0 & x & 0 & 0 & 0 & 0 & x & x \\
0 & 0 & x & x & 0 & 0 & 0 & 0 & 0 & x & x \\
\hline 0 & 0 & 0 & 0 & 0 & 0 & 0 & 0 & 0 & 0 & 0 \\
\hline x & 0 & 0 & 0 & 0 & 0 & 0 & 0 & 0 & 0 & 0 \\
x & x & 0 & 0 & 0 & 0 & 0 & 0 & 0 & 0 & 0 \\
0 & x & 0 & 0 & 0 & 0 & 0 & 0 & 0 & 0 & 0 \\
0 & 0 & x & x & x & 0 & 0 & 0 & 0 & 0 & x \\
0 & 0 & x & x & x & 0 & 0 & 0 & 0 & x & 0
\end{array}\right] .
$$

Proceeding with the analogous transformations as in the first subcase, until the row permutation step, we can obtain

$$
B_{0}=\left[\begin{array}{ll|llll|ll|ll|llll|ll}
x & x & x & x & x & x & x & x & x & x & x & x & x & x & 0 & 0 \\
0 & x & x & x & x & x & x & x & x & x & x & x & x & x & 0 & 0 \\
0 & 0 & x & x & x & x & x & x & x & x & x & x & x & x & 0 & 0 \\
0 & 0 & x & x & x & x & x & x & x & x & x & x & x & x & 0 & 0 \\
0 & 0 & x & x & x & x & x & x & x & x & x & x & x & x & 0 & 0 \\
\hline 0 & 0 & 0 & 0 & 0 & 0 & 0 & x & 0 & 0 & 0 & 0 & 0 & 0 & 0 & 0 \\
0 & 0 & 0 & 0 & 0 & 0 & x & x & 0 & 0 & 0 & 0 & 0 & 0 & 0 & 0 \\
\hline 0 & 0 & 0 & 0 & 0 & 0 & x & x & x & 0 & 0 & 0 & 0 & 0 & 0 & 0 \\
0 & 0 & 0 & 0 & 0 & 0 & x & x & x & x & 0 & 0 & 0 & 0 & 0 & 0 \\
0 & 0 & x & x & x & x & x & x & x & x & x & x & x & x & 0 & 0 \\
0 & 0 & x & x & x & x & x & x & x & x & x & x & x & x & 0 & 0
\end{array}\right]
$$


and

$$
B_{0} J B_{0}^{T}=\left[\begin{array}{ccccc|cc|cccc}
0 & 0 & 0 & 0 & 0 & 0 & 0 & x & x & 0 & 0 \\
0 & 0 & 0 & 0 & 0 & 0 & 0 & 0 & x & 0 & 0 \\
0 & 0 & 0 & x & x & 0 & 0 & 0 & 0 & x & x \\
0 & 0 & x & 0 & x & 0 & 0 & 0 & 0 & x & x \\
0 & 0 & x & x & 0 & 0 & 0 & 0 & 0 & x & x \\
\hline 0 & 0 & 0 & 0 & 0 & 0 & 0 & 0 & 0 & 0 & 0 \\
0 & 0 & 0 & 0 & 0 & 0 & 0 & 0 & 0 & 0 & 0 \\
\hline x & 0 & 0 & 0 & 0 & 0 & 0 & 0 & 0 & 0 & 0 \\
x & x & 0 & 0 & 0 & 0 & 0 & 0 & 0 & 0 & 0 \\
0 & 0 & x & x & x & 0 & 0 & 0 & 0 & 0 & x \\
0 & 0 & x & x & x & 0 & 0 & 0 & 0 & x & 0
\end{array}\right] .
$$

To maintain the block $B_{23}$ in upper triangular form and to maintain the condition $r \geq p$ we first perform a permutation to move the 5 th row of $B_{0}$ to the bottom and then perform another permutation to move row 6 to row 5 :

$$
B_{0}=\left[\begin{array}{ll|llll|ll|ll|llll|ll}
x & x & x & x & x & x & x & x & x & x & x & x & x & x & 0 & 0 \\
0 & x & x & x & x & x & x & x & x & x & x & x & x & x & 0 & 0 \\
0 & 0 & x & x & x & x & x & x & x & x & x & x & x & x & 0 & 0 \\
0 & 0 & x & x & x & x & x & x & x & x & x & x & x & x & 0 & 0 \\
\hline 0 & 0 & 0 & 0 & 0 & 0 & x & x & 0 & 0 & 0 & 0 & 0 & 0 & 0 & 0 \\
0 & 0 & 0 & 0 & 0 & 0 & 0 & x & 0 & 0 & 0 & 0 & 0 & 0 & 0 & 0 \\
\hline 0 & 0 & 0 & 0 & 0 & 0 & x & x & x & 0 & 0 & 0 & 0 & 0 & 0 & 0 \\
0 & 0 & 0 & 0 & 0 & 0 & x & x & x & x & 0 & 0 & 0 & 0 & 0 & 0 \\
0 & 0 & x & x & x & x & x & x & x & x & x & x & x & x & 0 & 0 \\
0 & 0 & x & x & x & x & x & x & x & x & x & x & x & x & 0 & 0 \\
0 & 0 & x & x & x & x & x & x & x & x & x & x & x & x & 0 & 0
\end{array}\right],
$$

and

$$
B_{0} J B_{0}^{T}=\left[\begin{array}{cccc|cc|ccccc}
0 & 0 & 0 & 0 & 0 & 0 & x & x & 0 & 0 & 0 \\
0 & 0 & 0 & 0 & 0 & 0 & 0 & x & 0 & 0 & 0 \\
0 & 0 & 0 & x & 0 & 0 & 0 & 0 & x & x & x \\
0 & 0 & x & 0 & 0 & 0 & 0 & 0 & x & x & x \\
\hline 0 & 0 & 0 & 0 & 0 & 0 & 0 & 0 & 0 & 0 & 0 \\
0 & 0 & 0 & 0 & 0 & 0 & 0 & 0 & 0 & 0 & 0 \\
\hline x & 0 & 0 & 0 & 0 & 0 & 0 & 0 & 0 & 0 & 0 \\
x & x & 0 & 0 & 0 & 0 & 0 & 0 & 0 & 0 & 0 \\
0 & 0 & x & x & 0 & 0 & 0 & 0 & 0 & x & x \\
0 & 0 & x & x & 0 & 0 & 0 & 0 & x & 0 & x \\
0 & 0 & x & x & 0 & 0 & 0 & 0 & x & x & 0
\end{array}\right] .
$$

Now $B_{0}$ and $B_{0} J B_{0}^{T}$ have the forms (4.5) and (4.6), respectively, but $p:=p-1$ and $q:=q+1$.

Because $B_{0}$ is of full row rank, the submatrix consisting of the third and fourth block rows in (4.5) must be of full row rank. Then both $B_{23}$ and the $(1,1)$ block of $B_{34}$ (in lower triangular form) must be nonsingular. Hence during the reductions no diagonal element in $B_{34}$ will be zero, and for deflation we need only to check the diagonal elements of $B_{11}$. In practice if $B_{11}(j, j)$ satisfies

$$
\left|B_{11}(j, j)\right|<\varepsilon\|B\|,
$$


we set it to zero and perform the deflation step described in case b.

Repeating the above reduction process, we will get (4.4).

We now perform a sequence of orthogonal symplectic transformations to transform (4.4) to (4.2). This is illustrated in the case when $p=2, q=3$, and $m=6$ :

$$
B_{0}=\left[\begin{array}{ll|l|lll|ll|l|lll}
x & x & x & x & x & x & x & x & x & 0 & 0 & 0 \\
0 & x & x & x & x & x & x & x & x & 0 & 0 & 0 \\
\hline 0 & 0 & 0 & x & x & x & 0 & 0 & 0 & 0 & 0 & 0 \\
0 & 0 & 0 & 0 & x & x & 0 & 0 & 0 & 0 & 0 & 0 \\
0 & 0 & 0 & 0 & 0 & x & 0 & 0 & 0 & 0 & 0 & 0 \\
\hline 0 & 0 & 0 & x & x & x & x & 0 & 0 & 0 & 0 & 0 \\
0 & 0 & 0 & x & x & x & x & x & 0 & 0 & 0 & 0
\end{array}\right] .
$$

Perform the symplectic Givens transformations $G_{1} \in \mathcal{G}_{3}^{s}(1,4), G_{2} \in \mathcal{G}_{3}^{s}(2,4)$ on the columns of $B_{0}$ to annihilate $B_{0}(6: 7,4)$ :

$$
B_{0}=\left[\begin{array}{ll|l|lll|ll|l|lll}
x & x & x & x & x & x & x & x & x & x & 0 & 0 \\
0 & x & x & x & x & x & x & x & x & x & 0 & 0 \\
\hline 0 & 0 & 0 & x & x & x & x & x & 0 & 0 & 0 & 0 \\
0 & 0 & 0 & 0 & x & x & 0 & 0 & 0 & 0 & 0 & 0 \\
0 & 0 & 0 & 0 & 0 & x & 0 & 0 & 0 & 0 & 0 & 0 \\
\hline 0 & 0 & 0 & 0 & x & x & x & 0 & 0 & 0 & 0 & 0 \\
0 & 0 & 0 & 0 & x & x & x & x & 0 & 0 & 0 & 0
\end{array}\right] .
$$

In the same way we can annihilate $B_{0}(6: 7,5)$ and $B_{0}(6: 7,6)$ :

$$
B_{0}=\left[\begin{array}{ll|l|lll|ll|l|lll}
x & x & x & x & x & x & x & x & x & x & x & x \\
0 & x & x & x & x & x & x & x & x & x & x & x \\
\hline 0 & 0 & 0 & x & x & x & x & x & 0 & 0 & 0 & 0 \\
0 & 0 & 0 & 0 & x & x & x & x & 0 & 0 & 0 & 0 \\
0 & 0 & 0 & 0 & 0 & x & x & x & 0 & 0 & 0 & 0 \\
\hline 0 & 0 & 0 & 0 & 0 & 0 & x & 0 & 0 & 0 & 0 & 0 \\
0 & 0 & 0 & 0 & 0 & 0 & x & x & 0 & 0 & 0 & 0
\end{array}\right] .
$$

Finally perform a symplectic permutation $P \in \mathcal{P}_{1}^{s}$ to move columns 3 and 9 to columns 6 and 12 , respectively. We have the form (4.2),

$$
B_{0}=\left[\begin{array}{ll|lll|l|ll|lll|l}
x & x & x & x & x & x & x & x & x & x & x & x \\
0 & x & x & x & x & x & x & x & x & x & x & x \\
\hline 0 & 0 & x & x & x & 0 & x & x & 0 & 0 & 0 & 0 \\
0 & 0 & 0 & x & x & 0 & x & x & 0 & 0 & 0 & 0 \\
0 & 0 & 0 & 0 & x & 0 & x & x & 0 & 0 & 0 & 0 \\
\hline 0 & 0 & 0 & 0 & 0 & 0 & x & 0 & 0 & 0 & 0 & 0 \\
0 & 0 & 0 & 0 & 0 & 0 & x & x & 0 & 0 & 0 & 0
\end{array}\right] .
$$

5. Error analysis. We only give an error analysis about the eigenvalues. We will provide the first order perturbation bound for a simple nonzero eigenvalue of $J B^{T} B$ or $B^{T} J B$. We will then use the perturbation bound to give the relative error bound for the computed eigenvalues. 
5.1. Perturbation about eigenvalues. All nonzero eigenvalues of $B J B^{T}$ and $J B B^{T}$ are purely imaginary and they are in conjugate pairs. For real perturbations the perturbation results for both eigenvalues in a conjugate pair are the same. For this reason in the following we consider only the eigenvalues $i \lambda$ with $\lambda>0$.

Suppose that $i \lambda$ is a simple nonzero eigenvalue of $B J B^{T}$ and $x$ is a corresponding unit norm eigenvector. Define another unit norm vector

$$
y=\frac{J B^{T} x}{\beta}
$$

with $\beta=\left\|J B^{T} x\right\|$. Premultiplying the equation by $J B^{T} B$, we have

$$
J B^{T} B y=i \lambda y .
$$

Hence $y$ is a unit norm eigenvector of $J B^{T} B$ corresponding to $i \lambda$. By using the conjugate transpose of the above equation we have

$$
(J y)^{*}\left(J B^{T} B\right)=i \lambda(J y)^{*} .
$$

So $J y$ is a unit norm left-eigenvector of $J B^{T} B$. The relation between $x, y$ is summarized as follows:

$$
B y=i \alpha x, \quad J B^{T} x=\beta y,
$$

where $\alpha=\frac{\lambda}{\beta}$. Taking the conjugate transpose of the second equation in (5.1) and postmultiplying it by $J y$,

$$
\beta y^{*} J y=x^{*} B y .
$$

Premultiplying the first equation in (5.1) by $x^{*}$,

$$
x^{*} B y=i \alpha .
$$

The reciprocal of the condition number of $i \lambda$ corresponding to the matrix $J B^{T} B$ is $\kappa=\left|(J y)^{*} y\right|=\left|y^{*} J y\right|$. Combining the above two equations,

$$
\kappa=\frac{\alpha}{\beta} .
$$

Since $\kappa \leq 1$ we have $\alpha \leq \beta$. Because $\lambda=\alpha \beta$ and $\beta=\left\|J B^{T} x\right\| \leq\|B\|$, we have

$$
\frac{\lambda}{\|B\|}<\alpha \leq \sqrt{\lambda} \leq \beta \leq\|B\| .
$$

The first order perturbation bound is given in the following lemma.

Lemma 5.1. Suppose that $i \lambda(\lambda>0)$ is a simple eigenvalue of $B J B^{T}$ and $J B^{T} B$, and $x, y$ are the corresponding unit norm eigenvectors with respect to $B J B^{T}$ and $J B^{T} B$, respectively, satisfying (5.1). Let $E$ be a real perturbation matrix and let $\hat{B}=B+E$. When $\|E\|$ is sufficiently small both matrices $\hat{B} J \hat{B}^{T}$ and $J \hat{B}^{T} \hat{B}$ have a purely imaginary eigenvalue $i \hat{\lambda}$ such that

$$
\left|\frac{i \hat{\lambda}-i \lambda}{i \lambda}\right|=\left|\frac{2 \operatorname{Im}\left(y^{*} E x\right)}{\alpha}\right|+O\left(\|E\|^{2}\right) \leq \frac{2\|E\|}{\alpha}+O\left(\|E\|^{2}\right) .
$$

Proof. The proof follows from the result in [4] for a formal matrix product. 
5.2. Error analysis. Again we consider only the case that $B J B^{T}$ is nonsingular. The general case can be analyzed in the same way. Because of rounding error, the algorithm in section 2 actually computes a block upper triangular matrix $R$ satisfying

$$
R=\left[\begin{array}{cc}
R_{1} & R_{2} \\
0 & R_{3}
\end{array}\right]=\left[\begin{array}{cccc}
R_{11} & R_{12} & R_{13} & R_{14} \\
0 & 0 & R_{23} & 0
\end{array}\right]=Q^{T}(B+E) U
$$

where $Q$ is orthogonal, $U$ is orthogonal symplectic, and $E$ is an error matrix satisfying $\|E\| \leq c \varepsilon\|B\|$ for some constant $c$. Suppose that $i \lambda(\lambda>0)$ is a simple eigenvalue of $B J B^{T}$ and $J B^{T} B$ with unit norm eigenvectors $x, y$ satisfying (5.1). When $\|E\|$ is sufficiently small by Lemma 5.1 there is an eigenvalue $i \hat{\lambda}$ of $R J R^{T}$ and $J R^{T} R$ such that

$$
\left|\frac{i \hat{\lambda}-i \lambda}{i \lambda}\right|=\frac{2\left|\operatorname{Im}\left(y^{*} E x\right)\right|}{\alpha}+O\left(\|E\|^{2}\right) \leq 2 c \varepsilon \frac{\|B\|}{\alpha}+O\left(\varepsilon^{2}\right) .
$$

However, the eigenvalues computed by the algorithm are $\pm i \delta_{1}, \ldots, \pm i \delta_{p}$, where $\delta_{1}, \ldots, \delta_{p}$ are the diagonal elements of $R_{11} R_{23}^{T}$. Because of rounding error the product $R J R^{T}$ is not exactly in the Schur-like form. By a straightforward analysis it satisfies

$$
R J R^{T}=\left[\begin{array}{cc}
0 & \Delta \\
-\Delta & 0
\end{array}\right]+\left[\begin{array}{cc}
F_{11} & F_{12} \\
-F_{12}^{T} & 0
\end{array}\right]=: \Gamma+F,
$$

where $\Delta=\operatorname{diag}\left(\delta_{1}, \ldots, \delta_{p}\right), F_{12}$ is strictly upper triangular, $F_{11}=-F_{11}^{T}$, and $\|F\| \leq$ $d \varepsilon\|B\|^{2}$ for some constant $d$. So the computed eigenvalues are the exact ones of $\Gamma$ and $i \hat{\lambda}$ in (5.4) is an eigenvalue of $\Gamma+F$. When $\|F\|$ is sufficiently small and we apply the perturbation result [16, sect. 4.2.2], [11, sect. 7.2.2] to $\Gamma+F$ for $i \hat{\lambda}$ there exists a corresponding eigenvalue of $\Gamma$, say $i \delta_{k}$, such that

$$
\left|i \hat{\lambda}-i \delta_{k}\right|=\left|z^{*} F z\right|+O\left(\|F\|^{2}\right),
$$

where $z=\frac{\sqrt{2}}{2}\left(e_{k}+i e_{p+k}\right)$ is the unit norm eigenvector of $i \delta_{k}$ (which is obvious from the structure of $\Gamma$ ). Because $F_{11}$ is real skew-symmetric and $F_{12}$ is strictly upper triangular,

$$
z^{*} F z=\frac{1}{2}\left(e_{k}^{*} F_{11} e_{k}+2 i e_{k}^{*} F_{12} e_{k}\right)=0 .
$$

Hence $\left|i \hat{\lambda}-i \delta_{k}\right|=O\left(\varepsilon^{2}\right)$. Combining it with (5.4) we have the error bound for $i \delta_{k}$,

$$
\left|\frac{i \delta_{k}-i \lambda}{i \lambda}\right|=\frac{2\left|\operatorname{Im}\left(y^{*} E x\right)\right|}{\alpha}+O\left(\varepsilon^{2}\right) \leq 2 c \varepsilon \frac{\|B\|}{\alpha}+O\left(\varepsilon^{2}\right) .
$$

For comparison we also give the error bounds for the eigenvalues computed by the numerically backward stable methods working on the explicit product $B J B^{T}$ or $J B^{T} B$. For both matrices explicitly forming the product will introduce an error matrix of order $\varepsilon\|B\|^{2}$. During the computations another error matrix will be introduced. Here for both matrices $J B^{T} B$ and $B J B^{T}$ we assume that the error matrix is of order $\varepsilon\|B\|^{2}$. (This is true for matrix $J B^{T} B$. But for matrix $B J B^{T}$ the order is $\varepsilon\left\|B J B^{T}\right\|$, which can be much smaller than $\varepsilon\|B\|^{2}$.) With standard perturbation analysis $[16$, sect. 4.2.2], [11, sect. 7.2.2] and by using the equality $\lambda=\alpha \beta$ and (5.2), for the simple eigenvalue $i \lambda$, the methods working on $B J B^{T}$ give an eigenvalue $i \hat{\lambda}_{s}$ satisfying

$$
\left|\frac{i \hat{\lambda}_{s}-i \lambda}{i \lambda}\right| \leq c_{s} \varepsilon \frac{\|B\|^{2}}{\lambda}+O\left(\varepsilon^{2}\right)=\left(c_{s} \varepsilon \frac{\|B\|}{\alpha}\right) \frac{\|B\|}{\beta}+O\left(\varepsilon^{2}\right)
$$


for some constant $c_{s}$. The methods working on $J B^{T} B$ give an eigenvalue $i \hat{\lambda}_{h}$ satisfying

$$
\left|\frac{i \hat{\lambda}_{h}-i \lambda}{i \lambda}\right| \leq c_{h} \varepsilon \frac{\|B\|^{2}}{\lambda \kappa}+O\left(\varepsilon^{2}\right)=\left(c_{h} \varepsilon \frac{\|B\|}{\alpha}\right) \frac{\|B\|}{\alpha}+O\left(\varepsilon^{2}\right)
$$

for some constant $c_{h}$. By (5.3),

$$
\frac{\|B\|}{\alpha} \geq \frac{\|B\|}{\beta} \geq 1
$$

So in general among three bounds (5.6) is the smallest and (5.8) is the biggest. When $\alpha$ or $\beta$ is small, $\|B\| / \alpha$ or $\|B\| / \beta$ can be much bigger than 1 . Since $\lambda=\alpha \beta$, this means that our method can compute tiny eigenvalues more accurately.

6. Numerical examples. We tested and compared the following numerical methods for computing the eigenvalues of the matrices $B J B^{T}$ and $J B^{T} B$.

$S S V D$. The SVD-like method presented in this paper;

CSVD. The SVD-like method applied to the matrix $L^{T}$, where $L$ is the Cholesky factor computed from the explicitly formed matrix $A:=B^{T} B$;

$S Q R$. QR method (bidiagonal-like reduction plus SVD) for $B J B^{T}$;

$J A C$. Jacobi method [15] for $B J B^{T}$;

$H A M$. Hamiltonian method $[2,3]$ for $J B^{T} B$.

All tests were done on a Dell PC with a Pentium 4 processor. All computations were performed in MATLAB version 6.1 with machine precision $\varepsilon \approx 2.22 \times 10^{-16}$.

Example 6.1.

$$
B=Q\left[\begin{array}{cc}
T^{5} & 0 \\
0 & T^{5}
\end{array}\right]
$$

where

$$
T=\left[\begin{array}{lllll}
2 & 1 & & & \\
1 & 2 & 1 & & \\
& 1 & 2 & 1 & \\
& & 1 & 2 & 1 \\
& & & 1 & 2
\end{array}\right],
$$

and $Q=5 I_{10}-e e^{T}$ with $e=\left[\begin{array}{lll}1 & \ldots & 1\end{array}\right]^{T}$. ( $Q / 5$ is a Householder matrix.) $\|B\|=$ $3.62 \times 10^{3},\left\|B J B^{T}\right\|=\left\|J B^{T} B\right\|=\|B\|^{2}=1.31 \times 10^{7}$.

This example is supposed to test the numerical behavior when no cancellation occurs in forming the product $B J B^{T}$. Note that

$$
B J B^{T}=Q\left[\begin{array}{cc}
0 & T^{10} \\
-T^{10} & 0
\end{array}\right] Q^{T}, \quad J B^{T} B=25\left[\begin{array}{cc}
0 & T^{10} \\
-T^{10} & 0
\end{array}\right] .
$$

Both matrices have exact eigenvalues $\pm i 25[2 \cos (k \pi / 12)]^{20}(k=1, \ldots, 5)$. Since all elements of $B$ are integers, no rounding error is introduced in forming the products $B J B^{T}$ and $J B^{T} B$.

The exact eigenvalues and the relative errors of computed eigenvalues are reported in Table 6.1.

In this example for each eigenvalue $i \lambda, \alpha=\beta=\sqrt{\lambda}$ and $\kappa=1$. From Table 6.1 it is clear that $S S V D$ gives eigenvalues with relative errors about $\frac{\|B\|}{\sqrt{\lambda}}$ times smaller 
TABLE 6.1

Example 6.1: Exact eigenvalues and relative errors.

\begin{tabular}{l|ccccc}
\hline Eigenvalue & rel $_{S S V D}$ & rel $_{C S V D}$ & rel $_{S Q R}$ & rel $_{J A C}$ & rel $_{H A M}$ \\
\hline $\pm i 4.77 \times 10^{-5}$ & $4.0 \times 10^{-12}$ & $4.7 \times 10^{-7}$ & $2.9 \times 10^{-6}$ & $1.2 \times 10^{-6}$ & $6.0 \times 10^{-6}$ \\
$\pm i 2.50 \times 10^{1}$ & $3.8 \times 10^{-15}$ & $4.2 \times 10^{-12}$ & $6.0 \times 10^{-12}$ & $2.9 \times 10^{-12}$ & $4.6 \times 10^{-12}$ \\
$\pm i 2.56 \times 10^{4}$ & $2.0 \times 10^{-15}$ & $2.0 \times 10^{-15}$ & $9.2 \times 10^{-15}$ & $3.6 \times 10^{-15}$ & $5.7 \times 10^{-15}$ \\
$\pm i 1.48 \times 10^{6}$ & $1.1 \times 10^{-15}$ & $1.4 \times 10^{-15}$ & $1.6 \times 10^{-15}$ & $1.6 \times 10^{-15}$ & $1.7 \times 10^{-15}$ \\
$\pm i 1.31 \times 10^{7}$ & $7.1 \times 10^{-16}$ & 0 & $1.4 \times 10^{-16}$ & $8.5 \times 10^{-16}$ & $5.7 \times 10^{-16}$ \\
\hline
\end{tabular}

TABLE 6.2

Example 6.1: Residuals and errors.

\begin{tabular}{l|cccc}
\hline & $S S V D$ & $C S V D$ & $S Q R$ & $J A C$ \\
\hline $\operatorname{err}_{S}$ & $4.6 \times 10^{-13}$ & $4.0 \times 10^{-13}$ & $2.9 \times 10^{-6}$ & $1.2 \times 10^{-6}$ \\
$\operatorname{res}_{B}$ & $1.3 \times 10^{-15}$ & - & $2.8 \times 10^{-16}$ & $8.9 \times 10^{-16}$ \\
$\operatorname{res}_{J}$ & $1.6 \times 10^{-15}$ & $1.3 \times 10^{-15}$ & $1.7 \times 10^{-16}$ & $3.2 \times 10^{-16}$ \\
$\operatorname{res}_{S C F}$ & $2.1 \times 10^{-13}$ & $1.9 \times 10^{-13}$ & $3.5 \times 10^{-11}$ & $9.1 \times 10^{-11}$ \\
\hline
\end{tabular}

than other methods. CSVD is basically the same as other methods. This is because computing the Cholesky factorization already introduced an error of order $O\left(\varepsilon\|B\|^{2}\right)$ to $A$.

We also computed the following quantities:

$$
\begin{aligned}
& \operatorname{err}_{S}=\max \left\{\left\|S J S^{T}-J\right\|,\left\|S^{T} J S-J\right\|\right\}, \quad r e s_{B}=\frac{\left\|Q D S^{-1}-B\right\|}{\|B\|}, \\
& r e s_{J A}=\frac{\left\|S\left(J D^{T} D\right) S^{-1}-J B^{T} B\right\|}{\left\|J B^{T} B\right\|}, \quad r e s_{S C F}=\frac{\left\|J D^{T} D-S^{-1}\left(J B^{T} B\right) S\right\|}{\left\|J D^{T} D\right\|},
\end{aligned}
$$

where $Q D S^{-1}$ is the SVD-like decomposition of $B$. These quantities are used to measure the accuracy of the symplectic matrix $S$, the residual of the SVD-like decomposition of $B$, the residual of the canonical form of $J B^{T} B$, and the accuracy of the eigenvectors, respectively. The matrices $S$ and $D$ are computed as follows. With $S S V D$ and $C S V D, S$ is computed by using $(2.5)$ and $D=\operatorname{diag}(\Sigma, \Sigma)$. With $S Q R$ and $J A C$, after obtaining the Schur-like form

$$
B J B^{T}=Q\left[\begin{array}{cc}
0 & \Delta \\
-\Delta & 0
\end{array}\right] Q^{T},
$$

we set $D=\operatorname{diag}(\sqrt{\Delta}, \sqrt{\Delta})$. Let $Z:=D^{-1} Q^{T} B$. Then $B=Q D Z$ and

$$
Z J Z^{T}=D^{-1} Q^{T} B J B^{T} Q D^{-1}=D^{-1}\left[\begin{array}{cc}
0 & \Delta \\
-\Delta & 0
\end{array}\right] D^{-1}=J .
$$

So we take $Z^{-1}$ as $S$. Since $Z$ is symplectic, $Z^{-1}=J Z^{T} J^{T}$. In practice we use the formula $S=J B^{T} Q D^{-1} J^{T}$ to compute $S$. The computed results are reported in Table 6.2. Both $S Q R$ and $J A C$ give slightly smaller residuals $r e s_{B}$ and res ${ }_{J A}$. But both $S S V D$ and $C S V D$ give much smaller $\operatorname{err}_{S}$, indicating that the matrix $S$ computed by $S S V D$ and $C S V D$ is more "symplectic."

Example 6.2.

$$
B=Q\left[\begin{array}{cc}
\Sigma & 0 \\
0 & \Sigma
\end{array}\right]\left(\left[\begin{array}{cc}
X & X \\
0 & X^{-1}
\end{array}\right] V^{T}\right)
$$


TABLE 6.3

Example 6.2: Exact eigenvalues and relative errors.

\begin{tabular}{c|ccccc}
\hline Eigenvalue & rel $_{S S V D}$ & rel $_{C S V D}$ & rel $_{S Q R}$ & rel $_{\text {JAC }}$ & rel $_{H A M}$ \\
\hline $\pm i$ & $6.9 \times 10^{-15}$ & $1.1 \times 10^{-10}$ & $2.7 \times 10^{-14}$ & $2.8 \times 10^{-14}$ & $5.8 \times 10^{-10}$ \\
$\pm 4 i$ & $1.2 \times 10^{-13}$ & $6.6 \times 10^{-9}$ & $8.0 \times 10^{-14}$ & $8.1 \times 10^{-14}$ & $1.5 \times 10^{-8}$ \\
$\pm 9 i$ & $5.3 \times 10^{-15}$ & $1.2 \times 10^{-13}$ & $2.0 \times 10^{-15}$ & $9.9 \times 10^{-16}$ & $2.2 \times 10^{-13}$ \\
$\pm 16 i$ & $2.8 \times 10^{-14}$ & $6.4 \times 10^{-13}$ & $3.4 \times 10^{-14}$ & $3.4 \times 10^{-14}$ & $4.9 \times 10^{-12}$ \\
$\pm 25 i$ & $1.6 \times 10^{-15}$ & $5.5 \times 10^{-12}$ & $1.3 \times 10^{-15}$ & $8.5 \times 10^{-16}$ & $1.5 \times 10^{-10}$ \\
\hline
\end{tabular}

TABLE 6.4

Example 6.2: Relative error bounds.

\begin{tabular}{c|ccc}
\hline Eigenvalue & $2 \varepsilon \frac{\|B\|}{\alpha}$ & $\varepsilon \frac{\|B\|^{2}}{\lambda}$ & $\varepsilon \frac{\|B\|^{2}}{\alpha^{2}}$ \\
\hline $\pm i$ & $2.2 \times 10^{-11}$ & $1.1 \times 10^{-10}$ & $5.6 \times 10^{-7}$ \\
$\pm 4 i$ & $1.1 \times 10^{-12}$ & $2.8 \times 10^{-11}$ & $5.6 \times 10^{-9}$ \\
$\pm 9 i$ & $1.3 \times 10^{-13}$ & $1.2 \times 10^{-12}$ & $1.7 \times 10^{-10}$ \\
$\pm 16 i$ & $7.8 \times 10^{-13}$ & $6.9 \times 10^{-12}$ & $1.1 \times 10^{-8}$ \\
$\pm 25 i$ & $6.3 \times 10^{-12}$ & $4.4 \times 10^{-12}$ & $1.1 \times 10^{-6}$ \\
\hline
\end{tabular}

where $\Sigma=\operatorname{diag}(5,4,3,2,1)$ and $X=\operatorname{diag}(100,10,1,0.1,0.01), Q$ is a random orthogonal matrix, and $V$ is a random orthogonal symplectic matrix. $\|B\|=7.07 \times 10^{2}$, $\|B\|^{2}=5.00 \times 10^{5}$.

This example is supposed to test the numerical behavior when big cancellation takes place in forming the product $B J B^{T}\left(\left\|B J B^{T}\right\|=25\right)$. The exact eigenvalues and the relative errors of the computed eigenvalues are reported in Table 6.3. For each eigenvalue $i \lambda$ the relative error bounds (5.6)-(5.8) are given in Table 6.4. (Here we set $c=c_{s}=c_{h}=1$.)

Because for the Hamiltonian matrix $J B^{T} B$ its eigenvalues have relatively big condition numbers, HAM gives less accurate eigenvalues. Again, CSVD also gives less accurate eigenvalues because of the Cholesky factorization. The other three methods compute the eigenvalues with the same accuracy, as predicted by the error bounds. The residuals of the decompositions and $\operatorname{err}_{S}, \operatorname{res}_{S C F}$ are reported in Table 6.5. In this example all these methods basically give the same results.

Example 6.3.

$$
B=Q\left[\begin{array}{ccc|ccc}
\Sigma & 0 & 0 & 0 & 0 & 0 \\
\hline 0 & I_{2} & 0 & 0 & 0 & 0 \\
\hline 0 & 0 & 0 & \Sigma & 0 & 0
\end{array}\right] U^{T}
$$

where $\Sigma=\operatorname{diag}\left(10^{-4}, 10^{-2}, 1,10^{2}\right), Q$ is a random orthogonal matrix, and $U$ is a $14 \times 14$ random orthogonal symplectic matrix. $\|B\|=10^{2}$ and $\|B\|^{2}=10^{4}$.

This example is supposed to test the numerical behavior when $B J B^{T}$ has (two) zero eigenvalues. The exact eigenvalues, the absolute errors for zero eigenvalues, and the relative errors for nonzero eigenvalues are reported in Table 6.6.

In this example for zero eigenvalues $S S V D$ gives the eigenvalues of order $\varepsilon$, while $S Q R, J A C$, and $H A M$ give answers about $\|B\|$ times bigger than $S S V D .{ }^{4}$ For nonzero eigenvalues, as in Example 6.1, SSVD gives the results with relative errors about $\frac{\|B\|}{\sqrt{\lambda}}$ times smaller than those of the other methods.

\footnotetext{
${ }^{4}$ The matrix $J B^{T} B$ actually has two additional $2 \times 2$ Jordan blocks corresponding to zero eigenvalues. The corresponding eigenvalues computed by $H A M$ are $\pm 8.37 \times 10^{-8} \pm 2.64 \times 10^{-7} i$.
} 
TABle 6.5

Example 6.2: Residuals and errors.

\begin{tabular}{l|cccc}
\hline & $S S V D$ & $C S V D$ & $S Q R$ & $J A C$ \\
\hline $\operatorname{err}_{S}$ & $8.8 \times 10^{-12}$ & $3.4 \times 10^{-11}$ & $3.2 \times 10^{-12}$ & $5.5 \times 10^{-13}$ \\
$\operatorname{res}_{B}$ & $1.2 \times 10^{-15}$ & - & $3.8 \times 10^{-16}$ & $1.6 \times 10^{-15}$ \\
$\operatorname{res}_{J A}$ & $1.3 \times 10^{-15}$ & $1.8 \times 10^{-15}$ & $2.1 \times 10^{-16}$ & $1.0 \times 10^{-15}$ \\
$\operatorname{res}_{S C F}$ & $3.1 \times 10^{-9}$ & $6.5 \times 10^{-10}$ & $3.1 \times 10^{-9}$ & $3.1 \times 10^{-9}$ \\
\hline
\end{tabular}

TABLE 6.6

Exact eigenvalues and errors for Example 6.3.

\begin{tabular}{c|cccc}
\hline Eigenvalue & rel $_{S S V D}$ & rel $_{S Q R}$ & rel $_{J A C}$ & rel $_{H A M}$ \\
\hline 0 (double) & $1.7 \times 10^{-15}$ & $1.1 \times 10^{-14}$ & $5.7 \times 10^{-14}$ & $1.5 \times 10^{-13}$ \\
$\pm i 10^{-8}$ & $1.9 \times 10^{-11}$ & $8.9 \times 10^{-6}$ & $1.3 \times 10^{-5}$ & $5.9 \times 10^{-6}$ \\
$\pm i 10^{-4}$ & $5.7 \times 10^{-13}$ & $1.7 \times 10^{-9}$ & $4.1 \times 10^{-11}$ & $7.5 \times 10^{-10}$ \\
$\pm i$ & $1.3 \times 10^{-15}$ & $1.1 \times 10^{-13}$ & $1.1 \times 10^{-14}$ & $2.1 \times 10^{-13}$ \\
$\pm i 10^{4}$ & $1.8 \times 10^{-16}$ & $1.8 \times 10^{-16}$ & $1.3 \times 10^{-15}$ & $3.6 \times 10^{-16}$ \\
\hline
\end{tabular}

In this example we did not test $C S V D$. Because in this case it is more complicated to compute the matrix $S$ by $S Q R$ and $J A C$, we did not compare the residuals and $e r r_{S}, \operatorname{res}_{S C F}$.

7. Conclusion. We have developed a numerical method to compute the SVDlike decomposition of a real matrix $B$. The method can be simply applied to compute the eigenvalues and canonical forms of the skew-symmetric matrix $B J B^{T}$ and the Hamiltonian matrix $J B^{T} B$. Unlike other numerical methods this method works only on the factor $B$. In this way the eigenvalues (particularly the small eigenvalues) of $B J B^{T}$ and $J B^{T} B$ can be computed more accurately. This has been demonstrated by the error bound and several numerical examples. The numerical examples also show that the symplectic matrix $S$ computed by the proposed method is more accurate.

Acknowledgment. The author gratefully acknowledges the anonymous reviewers for their valuable comments and suggestions on the first version of this paper.

\section{REFERENCES}

[1] P. Benner, R. Byers, V. Mehrmann, and H. Xu, Numerical computation of deflating subspaces of skew-Hamiltonian/Hamiltonian pencils, SIAM J. Matrix Anal. Appl., 24 (2002), pp. $165-190$.

[2] P. Benner, V. Mehrmann, And H. Xu, A new method for computing the stable invariant subspace of a real Hamiltonian matrix, J. Comput. Appl. Math., 86 (1997), pp. 17-43.

[3] P. Benner, V. Mehrmann, and H. Xu, A numerically stable, structure preserving method for computing the eigenvalues of real Hamiltonian or symplectic pencils, Numer. Math., 78 (1998), pp. 329-358.

[4] P. Benner, V. Mehrmann, And H. Xu, Perturbation analysis for the eigenvalue problem of a formal product of matrices, BIT, 42 (2002), pp. 1-43.

[5] A. Bojanczyk, G.H. Golub, and P. Van Dooren, The periodic Schur decomposition. Algorithms and applications, in Advanced Signal Processing Algorithms, Architectures, and Implementations III, Proc. SPIE 1770, SPIE, Bellingham, WA, 1992, pp. 31-42.

[6] P.A. Businger And G.H. Golub, Linear least squares solutions by Householder transformations, Numer. Math., 7 (1965), pp. 269-276.

[7] T. Chan, Rank revealing QR factorizations, Linear Algebra Appl., 88/89 (1987), pp. 67-82.

[8] R.J. Duffin, The Rayleigh-Ritz method for dissipative or gyroscopic systems, Quart. Appl. Math., 18 (1960), pp. 215-221.

[9] G. Golub AND W. Kahan, Calculating the singular values and pseudo-inverse of a matrix, SIAM J. Numer. Anal., 2 (1965), pp. 205-224. 
[10] G. Golub, K. Sølna, And P. Van Dooren, Computing the SVD of a general matrix product/quotient, SIAM J. Matrix Anal. Appl., 22 (2000), pp. 1-19.

[11] G. Golub and C.F. Van Loan, Matrix Computations, 3rd ed., The Johns Hopkins University Press, Baltimore, MD, 1996.

[12] J.J. Hench And A.J. Laub, Numerical solution of the discrete-time periodic Riccati equation, IEEE Trans. Automat. Control, 39 (1994), pp. 1197-1210.

[13] P. Lancaster, Lambda-Matrices and Vibrating Systems, Pergamon Press, Oxford, UK, 1966.

[14] C.B. Moler and G.W. Stewart, An algorithm for generalized matrix eigenvalue problems, SIAM J. Numer. Anal., 10 (1973), pp. 241-256.

[15] M.H.C. PAaRdeKoOPer, An eigenvalue algorithm for skew-symmetric matrices, Numer. Math., 17 (1971), pp. 189-202.

[16] G.W. Stewart and J.-G. Sun, Matrix Perturbation Theory, Academic Press, New York, 1990.

[17] F. Tisseur and K. Meerbergen, The quadratic eigenvalue problem, SIAM Rev., 43 (2001), pp. 235-286.

[18] H. XU, An SVD-like matrix decomposition and its applications, Linear Algebra Appl., 368 (2003), pp. 1-24.

[19] V.A. Yakubovich and V.M. Starzhinskit, Linear Differential Equations with Periodic Coefficients, Vols. 1 and 2, Halstead, New York, Toronto, 1975. 